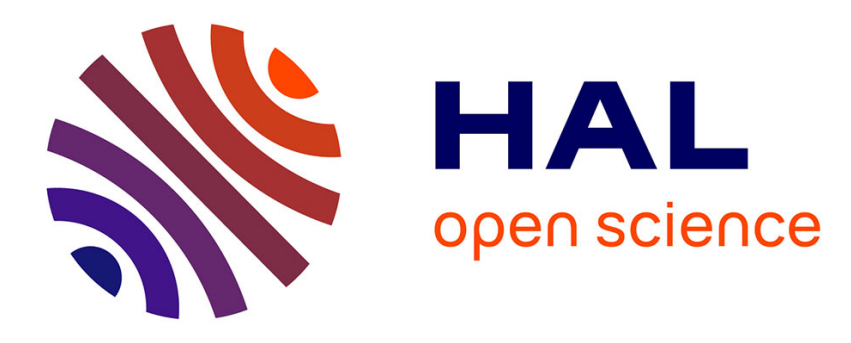

\title{
Hydrogen effects on the plasticity of face centred cubic (fcc) crystals
}

David Delafosse

\section{To cite this version:}

David Delafosse. Hydrogen effects on the plasticity of face centred cubic (fcc) crystals. Richard P. Gangloff and Brian P. Somerday. Gaseous hydrogen embrittlement of materials in energy technologies Volume 2, Woodhead Publishing Ltd., pp.247-285, 2012, 978-0-85709-536-7. 10.1533/9780857095374.2.247 . hal-00989385

\section{HAL Id: hal-00989385 https://hal.science/hal-00989385}

Submitted on 11 May 2014

HAL is a multi-disciplinary open access archive for the deposit and dissemination of scientific research documents, whether they are published or not. The documents may come from teaching and research institutions in France or abroad, or from public or private research centers.
L'archive ouverte pluridisciplinaire HAL, est destinée au dépôt et à la diffusion de documents scientifiques de niveau recherche, publiés ou non, émanant des établissements d'enseignement et de recherche français ou étrangers, des laboratoires publics ou privés. 


\title{
Hydrogen effects on the plasticity of face-
}

\section{centered cubic crystals}

\section{David Delafosse}

Ecole Nationale supérieure des Mines de Saint-Etienne, Laboratoire Claude Goux UMR CNRS 5146, 158 Cours Fauriel, 42023 Saint-Etienne Cedex 2 - France

david.delafosse@emse.fr

Keywords: hydrogen, discrete dislocations, plasticity, ageing, trapping, modelling, single crystals

\begin{abstract}
The dynamic interactions between solid solution hydrogen atoms and dislocations in face centred cubic crystals are considered. Using strain ageing experiments, the strength of the elastic binding between dislocations and solutes atmospheres is measured. These results support the Linearized Hydrogen Elasticity framework for modelling Hydrogen - dislocations interactions. It is used to derive quantitative estimates of Hydrogen effects on dislocation properties. Consequences are predicted on the Franck-Read mechanism and the cross-slip probability of screw dislocations. They are compared to the tensile response of Nickel single crystals. Cross-slip is the mechanism whose alteration by Hydrogen has the most important consequences on deformation. Effects on Hydrogen-induced damage are discussed and possible extensions to other classes of metals and alloys are outlined.
\end{abstract}




\section{Contents}

1. Introduction and scope

2. Study of dynamic interactions and elastic binding by Static Strain Ageing

2.1. Background

2.1.1. Discontinuous motion of dislocation in f.c.c. alloys and ageing mechanism

2.1.2 DSA and SSA

2.2. SSA measurements in Hydrogenated Nickel and Ni-Cr

2.2.1. Materials preparation and experimental setup

2.2.2. Measurements

2.3 Summary: Elastic binding and solute drag

3. Modelling in the framework of the elastic theory of discrete dislocations

3.1. Theoretical background in linear elasticity

3.1.1. Linear-elastic theory of the equilibrium between interstitial solid solution atoms and the host metal lattice

3.1.2. "Elastic screening of stress centres"

3.2. Hydrogen effects on dislocation properties

3.2.1. H-modified self-stress field

3.2.2. Screening of dislocation pair interactions

3.2.3. Line energy and line tension

3.3. Consequences on plasticity mechanisms

3.3.1. Multiplication by Franck-Read Sources

3.3.1.1. Critical activation stress

3.3.1.2. Isotropic loop expansion

3.3.2. Cross-slip probability of screw dislocations

3.3.2.1. H-effects on the dissociated configuration

3.3.2.2. H-effects on the recombination work and cross-slip probability

3.5. Summary of modelling results

4. Experiments on f.c.c. single crystals oriented for single glide

4.1. Cyclic plasticity

4.2. Monotonous tension

4.2.1. Tensile behaviour

4.2.2. TEM observations

5. Review of main conclusions

6. Directions for further developments

7. References

Figures 


\section{Introduction and scope}

Experimental evidences of hydrogen affecting the flow and fracture behaviour of metallic materials are numerous. They are thoroughly reviewed by Robertson et al. in their chapter on the influence of hydrogen on the behaviour of dislocations. Among the pioneering experimental works on the effects of solute Hydrogen on plasticity, those carried out by Smith and co-workers at Cambridge University in the 60's are the most relevant to the cases investigated in this chapter (Wilcox \& Smith 1964; Windle \& Smith 1968). The present chapter focuses on the case of mobile solid solution hydrogen in austenitic and face-centred cubic metals and alloys, and develops the implications of the concept of "Hydrogen-induced softening" introduced at the University of Illinois by Birnbaum and co-workers (Birnbaum, 1994). The "Linearized Hydrogen Elasticity" framework proposed by Sofronis (Sofronis 1995) to support the Hydrogen Enhanced Localized Plasticity mechanism (Birnbaum \& Sofronis 1994) is particularly relevant to the case of non-hydride forming systems that display a relatively large solubility and low mobility of solid solution hydrogen, which is the case of face-centred cubic (f.c.c.) metals and alloys, such as austenitic stainless steels and Nickel-based alloys. In these materials, the flow properties of crystals are governed by the collective behaviour of dislocations and have to be modelled at the scale of the elastic theory of crystal defects. Based on Larché \& Cahn's theory of chemo-elasticity (Larche \& Cahn 1985), Linearized Hydrogen Elasticity has the same scope as the linear elastic theory of crystal defects. It is supported by unique experimental observations obtained in the environmental TEM at the University of Illinois (P.J. Ferreira et al. 1998; Sofronis \& I.M. Robertson 2002). In this chapter we will see that, provided a few reasonable assumptions are made, this approach may be elaborated upon to derive simple expressions that render the - mainly dilatational - elastic interactions between solute hydrogen atoms and dislocations and use these to predict hydrogen effects on elementary plasticity mechanisms.

We first describe evidences of dynamic interactions between solid solution hydrogen and mobile dislocations in f.c.c. metals. Interactions between solute hydrogen and dislocations may be quantified using static strain ageing measurements at cryogenic temperatures on hydrogenated 
samples. We detail these experiments and the underlying mechanisms because they give a physical insight to the dynamics of hydrogen - dislocation interactions in f.c.c. alloys.

The second part of this chapter is dedicated to the modelling of elastic interactions between mobile hydrogen in solid solution and individual dislocations. Revisiting traditional results of dislocation theory, we elaborate on H-effects on elementary plasticity mechanisms.

The third part of the chapter goes back to experiments and attempts to validate the modelling results by investigating the stress-strain response of hydrogen-containing nickel single crystals. These measurement, supplemented by Transmission Electron Microscopy observations of dislocation microstructures at different levels of strain, point out the effect of hydrogen on the cross-slip of screw dislocations as being the most influential contribution to the flow of H-containing f.c.c. crystals.

The modelling and experimental results are finally summarized and possible consequences on the fracture behaviour of f.c.c. alloys in the presence of hydrogen are discussed.

\section{Study of dynamic interactions and elastic binding by Static Strain Ageing}

The first level of interactions between plasticity and hydrogen atoms in solid solution is the trapping and transport of mobile hydrogen atoms in the elastic field and core of dislocations. Autoradiography of deformed samples containing tritium and tritium desorption measurements by $\beta$ counting during plastic straining provide direct experimental evidences of this effect (Brass 1996; Chene \& Brass 1999; Chene et al. 2007). We are describing here an alternative experimental approach. Although indirect, this procedure is easier to carry out because it does not require tritiated samples. It gives a physical insight to the often quoted phenomenon of 'hydrogen transport by dislocations' (Bastien \& Azou, 1951; Donovan, 1973; Chene \& Brass, 1999). This approach is based on the phenomena of static and dynamic strain ageing, which appear in dilute solid solution alloys deformed in a strain rate / temperature domain where the mobility of dislocations and that of solute atoms are of the same order of magnitude. Dynamic Strain Ageing (DSA) is well documented in many dilute alloys such as, e.g.: Al-Mg around room temperature (Kubin et al., 1988), Fe-C around $300^{\circ} \mathrm{C}$ (De, 1999), or austenitic alloys around $350^{\circ} \mathrm{C}$ (Samuel et al., 1988). Its most spectacular manifestation is the occurrence of Portevin-Le Chatelier (PLC) instabilities, which 
occur when the net Strain Rate Sensitivity (SRS) of the flow stress (i.e.: the viscosity) becomes negative. In such a situation, the system (machine + tensile specimen) is unstable and sequences of load drops appear on the stress-strain curve, accompanied by series of localised deformation bands along the gauge length of the specimen (Chihab et al. 1987; Kubin et al. 1988; Varadhan et al. 2009).

PLC instabilities in the Ni-H system were first reported by Wilcox and Smith at cryogenic temperatures (Wilcox \& Smith 1964). Combette and Grilhé later conducted a thorough study of the conditions for the appearance of such instabilities in hydrogenated nickel, which allowed relating them unambiguously to DSA (Combette \& Grilhe 1970). More recently, Girardin (Girardin 2004) showed that the "two dislocation densities" model proposed by Kubin and Estrin (Kubin \& Estrin 1990) for Al-Mg at room temperature could be adapted to hydrogenated nickel around $-140^{\circ} \mathrm{C}$ and used for the quantitative assessment of the experimental data presented in this section. We first recall the basic mechanisms of dynamic interactions between solutes and dislocations that govern DSA. We then present the measurements carried out in high purity hydrogenated nickel and Ni$16 \mathrm{Cr}$ binary alloy and conclude on the dynamics of $\mathrm{H}$-plasticity interactions at room temperature in these materials.

\subsection{Background}

\subsubsection{Discontinuous motion of dislocation in f.c.c. alloys and ageing mechanism}

In f.c.c. alloys, the lattice friction is almost negligible and the motion of dislocations can be pictured as a succession of instantaneous jumps between forest obstacles. The waiting time between two thermally activated jumps is denoted $t_{w}$ and, under the classical assumption that the mean free path between forest obstacles scales like the square root of the density of forest dislocations (i.e.: dislocations belonging to other slip systems that intersect the slip plane of a mobile dislocation), one can rewrite Orowan's law as:

$\dot{\varepsilon}_{p}=\frac{b \rho_{m} \rho_{f}^{1 / 2}}{t_{w}}$ 
with $\dot{\varepsilon}_{p}$ the plastic strain rate, $\rho_{m}$ and $\rho_{f}$ the mobile and forest dislocation densities respectively, and $b$ the magnitude of the Bürger's vector of perfect dislocations. In the presence of segregating solutes, mobiles dislocations are "aged" (i.e.: pinned) during their waiting time between two thermally activated jumps. The sequence may be depicted as in figure 1 . It is worth noting that, if taking place in a uniform remote concentration field, this process does not require the transport of solute atoms over long distances because the ageing of a dislocation stems from solute segregation from the local uniform concentration around the current position. In the case of hydrogen, this may induce differences in ageing kinetics as a function of the boundary conditions for diffusion (e.g.: uniformly pre-charged samples vs. hydrogen uptake from a surface or a crack-tip).

\subsubsection{DSA and SSA}

The ageing time $t_{a}$ should be lower or equal to the waiting time $t_{w}$ because the ageing process for DSA does not adjusts instantaneously to changes in dislocation dynamics (McCormick 1988). We will assume that these times are equal and that both quantities are interchangeable in subsequent equations. Kubin \& Estrin's model assumes an exponential kinetic of ageing:

$f\left(t_{a}\right)=f_{0}\left[1-e^{\left(-t_{a} / \tau\right)^{\frac{2}{3}}}\right]$

where $t_{a}$ is the ageing time, $f$ is the pinning force, $f_{0}=\lim _{t_{a} \rightarrow \infty}(f)$ is the saturated value of this force, and $\tau$ the time constant of ageing. Saturation corresponds to a situation where the distribution of solutes is "in equilibrium with local stress gradients" (Sofronis 1995) and recalled in section 3.1.1. From diffusion calculations (Chateau et al. 2002), this saturation time is of the order of $10^{-3} \mathrm{~s}$ for hydrogen in an austenitic stainless steel at $120^{\circ} \mathrm{C}$ (i.e.: in boiling concentrated $\mathrm{MgCl}_{2}$, an environment known to trigger dramatic hydrogen embrittlement effects in austenitic stainless steels).

Dynamic Strain Ageing occurs in a domain where the waiting time is comparable to the ageing time constant $\tau$. In such a situation, it can be seen from equation 1 that a local increase of the plastic strain rate will decrease the waiting time $t_{w}$ and the available time for ageing $t_{a}$. From equation 2 , in a domain where $t_{a} \sim \tau$, this decrease will cause a decrease of the pinning force: we are indeed in the 
presence of a negative contribution to the Strain Rate Sensitivity (SRS) of the flow stress, as defined by equation 3 , for an increment of plastic strain rate $\Delta \dot{\varepsilon}_{p}$ :

$\operatorname{SRS}\left(\varepsilon_{p}\right)=\Delta \sigma / \Delta \ln \left(\dot{\varepsilon_{p}}\right)$

If the magnitude of this negative contribution becomes large enough to cancel the (positive) athermal contribution, the net SRS turns negative and plastic instabilities appear. The domain of PLC instabilities is thus a sub-domain of the DSA domain. In the Kocks-Mecking approach (Kocks \& Mecking, 2003); a deformation microstructure is entirely described by the two dislocation densities $\rho_{m}$ and $\rho_{f}$. At a given strain, these quantities are given and the condition $t_{w} \approx t_{a}$ is controlled by the strain rate only. On the other hand, $\tau$ is set by the temperature. At a given strain rate, the temperature at which the SRS is minimum corresponds to the condition $\tau \approx t_{a} \approx t_{w}$. This can be conveniently expressed by stating that "the mobility of solutes and that of dislocations are identical", keeping in mind the fact that the latter is an apparent one, given by the ratio of the average flight distance between obstacles over the waiting time. Figure 2 shows a minimum of the SRS in both hydrogenated $\mathrm{Ni}$ and Ni-Cr, evidencing DSA in both systems, but, for our charging conditions, only $\mathrm{H}$-containing $\mathrm{Ni}$ displays negative values between $-120^{\circ} \mathrm{C}$ and $-40^{\circ} \mathrm{C}$ and associated PLC instabilities (Girardin \& Delafosse, 2004).

We are interested in two situations, just outside the DSA domain:

- At higher temperatures / lower strain rates $\left(\tau \gg \tau_{\mathrm{w}}\right)$, dislocations are constantly aged to saturation during monotonous straining. The repeated unpinning and re-aging of mobile dislocations during deformation results in a nearly constant positive contribution to the flow stress commonly denoted as the "drag of solute atmospheres". This situation is that of hydrogenated Nickel at room temperature and above. It is worth noting that this temperature domain is also that where the "hydrogen-induced softening" phenomenon (Birnbaum, 1994) is expected to occur. Section 4 will discuss in details the different contributions of solute hydrogen to the flow stress of single crystals.

- At lower temperatures / higher strain rates $\left(\tau<<\tau_{\mathrm{w}}\right)$, solute diffusion is too slow for dislocations to be aged significantly during their waiting time. Dislocations move in a uniform solid solution. 
Static Strain Ageing (SSA) experiments can be carried out in this second domain, temperatures lower than that of DSA for the strain rate of interest. If deformation is abruptly interrupted and the load kept constant during a relatively long holding time $t_{h}$,provided there is no significant relaxation during this time at the temperature of interest, one may assume $t_{h} \approx t_{a} \approx t_{w}$. We now have a direct control over the ageing time $t_{a}$, through the holding time at near-zero stress $t_{h}$. Upon reloading at the initial strain rate, a transient peak of the flow stress is observed (figure 3 ). The amplitude of this peak is a fraction of $f_{0}$. The "missing" fraction corresponds to the early unpinning of dislocations during reloading; before the peak stress is reached (reloading is conducted at a finite strain rate). Using Kubin and Estrin model (Kubin \& Estrin 1990; Kubin et al. 1992), this fraction can be estimated from the duration of the transient phase.

\subsection{SSA measurements in Hydrogenated Nickel and $\mathrm{Ni}-\mathrm{Cr}$}

\subsubsection{Materials preparation and experimental setup}

We describe here the sample preparation procedure for SSA measurements. The hydrogen charging procedure described below is the same for the plasticity experiments presented in section 5.4, although the samples are different.

Cylindrical tensile specimens with a $2.5 \mathrm{~mm}$ radius and a $25 \mathrm{~mm}$ gauge length were cut in polycrystalline commercial purity nickel and in a binary nickel - 16 wt. \% chromium alloy elaborated from it. Both materials contained less than 25 wt. ppm P, less than 15 wt. ppm C, and less than 10 wt. ppm $\mathrm{O}, \mathrm{N}$ and $\mathrm{S}$. They were solution treated $40 \mathrm{~min}$ at $800^{\circ} \mathrm{C}$ and water quenched. Samples were thermally hydrogenated in an autoclave in order to get two hydrogen levels. The "high" level of hydrogen charging was achieved by a 48 hours exposure to a $15 \mathrm{MPa}$ pressure of $\mathrm{H}_{2}$ gas at $450^{\circ} \mathrm{C}$ and resulted in a 1900 at. ppm concentration both in pure nickel and in Ni-16Cr. The "low" level was obtained under $10 \mathrm{MPa}$ pressure of $\mathrm{H}_{2}$ gas at $350^{\circ} \mathrm{C}$, giving a 1500 at. ppm hydrogen concentration for both metals. After exposure, the specimens were air-cooled during 2 min, water-quenched, and further cooled in liquid nitrogen. They were stored in a liquid $\mathrm{N}_{2} \operatorname{tank}$ before mounting and mechanical testing. For comparison purposes, reference (non-hydrogenated) samples were exposed to purified argon at $450^{\circ} \mathrm{C}$ during 48 hours, in order to account for a possible recovery of the dislocation density during thermal hydrogen charging. Tensile specimens were 
tested in a servo hydraulic machine. Temperature control was achieved within $+/-1^{\circ}$ by means of a climatic chamber with heating walls and a PID-controlled servo valve ejecting a liquid $\mathrm{N}_{2}$ spray.

\subsubsection{Measurements}

In Nickel, monotonous tensile loading around $-70^{\circ} \mathrm{C}$ at a strain rate of $10^{-2} \mathrm{~s}^{-1}$ produces "type C" PLC instabilities (see (Chihab et al. 1987) for a description of the three different types of PLC instabilities). Figure 2 displays the measured SRS in both materials. In hydrogenated Nickel, the SRS is negative down to $-130^{\circ} \mathrm{C}$, whereas $\mathrm{Ni}-16 \mathrm{Cr}$ displays a minimum around $-60^{\circ} \mathrm{C}$ associated to DSA, but no domain of PLC instabilities. Following the argument above, SSA measurements are carried out at $-140^{\circ} \mathrm{C}$ in Nickel, and $-100^{\circ} \mathrm{C}$ in $\mathrm{Ni}-16 \mathrm{Cr}$ with a base strain rate of $10^{-2} \mathrm{~s}^{-1}$. The measurement procedure is the following:

i. After pre-loading at $50 \%$ of the yield stress and temperature stabilisation, tensile testing starts at a constant engineering strain rate $\left(10^{-2} \mathrm{~s}^{-1}\right)$, up to $7 \%$ of plastic strain.

ii. At $\varepsilon_{p}=7 \%$, the sample is unloaded down to $5 \mathrm{MPa}$ and the testing rig is switched to load-control during a holding time $t_{h}=10$ to $3.10^{4} \mathrm{~s}$. Under such a low load, no significant plastic relaxation occurs, and we assume $t_{h}=t_{a} \sim t_{w}$.

iii. Tensile straining resumes at the initial strain rate. A transient peak of the flow stress is recorded, which corresponds to the adjustment of the system to the imposed change in strain rate, until a new permanent regime is reached. The peak stress $\Delta \sigma$ is measured according to the procedure recommended by (Combette \& Grilhe 1970) (figure 3).

Figures 4.a and 4.b, respectively, give the value of the measured stress peaks $\Delta \sigma$ as a function of the holding time $t_{h}$ in hydrogenated Nickel and $\mathrm{Ni}-16 \mathrm{Cr}$, respectively, for both hydrogen contents. These curves exhibit a behaviour consistent with the modelling of static strain ageing by the Kubin and Estrin (Kubin et al. 1992) "dislocation model" and with the work of De et al. on ultra low carbon bake-hardening steel (De 1999). $\Delta \sigma$ incubates at a non-zero value for short holding times, then grows with $t_{h}$ and eventually saturates to $\Delta \sigma_{\max }$ at long times. The non-zero value at short ageing times corresponds to the extra stress necessary to accommodate the sharp change in plastic strain rate upon reloading, in the absence of ageing effects. Saturation is clearly reached after $10^{4} \mathrm{~s}$ in Ni-16Cr. The saturation value is an increasing function of the hydrogen content. For the 1900 at. 
ppm $\mathrm{H}$ content, the saturation is about $16 \mathrm{MPa}$ whereas it is about $13 \mathrm{MPa}$ for a concentration of 1500 at. ppm. Hydrogenated nickel displays the same behaviour as the alloy, but the complete saturation is not reached for an ageing time of 8 hours at $-140^{\circ} \mathrm{C}$. Although our experimental setup did not allow checking it at longer holding times, we expect that saturation will be reached after $10^{5}$ $\mathrm{s}$ and will be slightly higher than for the alloy. $f_{0}$ is the saturated value of $\Delta \sigma$ that can be produced by the ageing mechanism. It measures the maximum pining stress between solute hydrogen and mobile dislocations during straining.

\subsection{Summary: Elastic binding and solute drag}

In addition to providing an insight to the dynamics of the interactions between solute hydrogen and mobile dislocation, as they are described in section 2.1.1., these measurements allow drawing two important conclusions:

- At the cryogenic temperatures investigated, "deep trapping" sites such as dislocation cores are expected to be saturated at much lower concentration levels than those studied here. The increase of pinning force when going from 1500 to 1900 at.ppm is thus attributed to the "elastic" trapping of hydrogen in the hydrostatic stress field of dislocations. Although indirect, the experimental method presented here provides an access to this effect, predicted by linear elasticity (see section 3).

- At "high" (room) temperature, where mobile dislocations are continuously aged during straining, the "solute drag" force corresponds to a constant hardening contribution to the flow stress of the same order of magnitude (15 to $20 \mathrm{MPa}$ ). This solute drag contribution to the flow stress was also identified through direct measurements at room temperature (see section 4.2.1.)

These results support the modelling approach presented in section 3, and will be useful to interpret some of the experimental results on the effects of hydrogen on the stage I deformation of nickel single crystals (section 4) 


\section{Modelling in the framework of the elastic theory of discrete dislocations}

The previous section provided a detailed experimental account of the elastic binding between solute atmospheres of hydrogen atoms and mobile dislocations. The most important practical conclusion for modelling is that, for "ordinary" strain rates at room temperature and above, dislocations are continuously aged to saturation during straining. This section on modelling strongly relies on this experimental finding: calculations of the elastic effect of hydrogen solutes on dislocation properties presented here are based on the assumption of a steady state distribution of solutes in equilibrium with local stress gradients. We first recall the theoretical background of the "Linearized Hydrogen Elasticity" theory, as it was introduced by Sofronis (Sofronis 1995). Indeed, section 3.1 is summarizing the modelling framework formalised by Sofronis. Similarly, section 3.1.2. essentially consists in reformulating the concept of "elastic screening of stress centres" proposed by Sofronis and Birnbaum (Sofronis \& H. K. Birnbaum 1995) in a form adapted to the framework of the elastic theory of discrete dislocations. We then elaborate on the effects of solute hydrogen on the elementary properties of dislocations and on elementary plasticity mechanisms that involve simple dislocation configurations.

\subsection{Theoretical background in linear elasticity}

Solid solution hydrogen atoms interact with lattice defects via an isotropic dilatation of the host lattice. This is expressed by hydrogen's partial molar volume, i.e.: the unconstrained volume dilatation of the metal containing one mole of hydrogen. A typical value is $\mathrm{V}^{*}=2 \mathrm{~cm}^{3} / \mathrm{mol}$ for f.c.c. materials (Chene \& Brass 1990). An isochore introduction of hydrogen into a lattice creates a hydrostatic compression stress. Thereby, the mean hydrostatic stress affects the hydrogen solubility in the host metal, and hydrostatic stress gradients affect hydrogen diffusion and distribution. Larché and Cahn (Larche \& Cahn 1985) studied these interactions by linearising the equilibrium equations around the average uniform concentration in the dilute solutions approximation. This approach, further rationalised by Sofronis (Sofronis 1995) for the case of interstitial hydrogen, allows for the determination of the strain field induced by the local hydrogen concentration at steady state. From these results, it is possible to evaluate the effect of hydrogen on the pair interactions between 
dislocations and on the self-stress field of a dislocation line in an isotropic crystal. We first recall the main results of this analysis, before applying it to the elastic theory of discrete dislocations.

\subsubsection{Linear-elastic theory of the equilibrium between interstitial solid solution atoms and the host metal lattice}

Sofronis demonstrated that in the presence of an average uniform volume concentration $\mathrm{C}_{0}$ (in at.m

${ }^{3}$ ); this solute concentration is to be taken as the reference state for chemical potentials. The lattice distortion resulting from an excess concentration of solid solution atoms is expressed by $\boldsymbol{H}$, the dilatation tensor associated to one solute atom. In the absence of applied stress, the strain $\varepsilon^{s s}$ associated to a local concentration $C$ of the solid solution is:

$\varepsilon_{i j}^{S S}=\boldsymbol{H}_{i j}\left(C-C_{0}\right)$

Under a local stress tensor $\sigma$, the strain tensor becomes:

$\varepsilon_{i j}=\boldsymbol{K}_{i j k l} \sigma_{k l}+\boldsymbol{H}_{i j}\left(C-C_{0}\right)$

with $\boldsymbol{K}$ the compliance tensor at the nominal concentration $C_{0}$. The chemical potential $\mu\left(\sigma_{-} i j, T, C\right)$ corresponds to the isothermal work necessary to bring an atom from infinity to its normal lattice position (Larche \& Cahn 1985):

$\mu\left(\sigma_{i j}, T, C\right)=\mu_{0}+k T \ln \left(\frac{C}{C_{0}}\right)-\boldsymbol{H}_{i j} \sigma_{i j}$

with $\mu_{0}$ the chemical potential in the reference state, $k$ Boltzmann's constant and $T$ the absolute temperature. From this expression, hydrostatic tensile stresses lower the chemical potential of solute hydrogen. Chemical potential gradients cause a flow of hydrogen, which is governed by the first Fick's law:

$\vec{J}=-\frac{D_{H} C}{k T} \vec{\nabla} \mu$

with $D_{H}$, the lattice diffusion coefficient at temperature $T$, and $k$, Boltzmann's constant. From equations (6) and (7), the second Fick's law becomes: 


$$
\frac{\partial C}{\partial t}=-\vec{\nabla} \cdot \vec{J}=D_{H} \nabla^{2} C-\frac{D C_{0}}{k T} \nabla^{2}\left(\boldsymbol{H}_{i j} \sigma_{i j}\right)
$$

At steady state, the equilibrium concentration can be defined when the flow defined by equation (7) is uniformly zero, i.e.: for a zero gradient of the chemical potential (Sofronis 1995). Integrating the void flow condition, one obtains the concentration $C$ "in equilibrium with the local stress gradient", as assumed in the analysis of strain ageing experiments in section 2.3:

$$
C-C_{0}=\frac{H_{i j} \sigma_{i j}}{k T}
$$

This condition will be assumed in the remainder of the modelling results presented in this chapter. For room temperature deformation at "ordinary" strain rates in f.c.c. austenitic materials, it was justified by the strain ageing measurements (\$2.3.).

\subsection{2. "Elastic screening of stress centres"}

Because of the induced lattice distortion, solid solution atoms affect in turn the compliance tensor, which is obtained by substituting the steady state condition (equation. 9) in the constitutive equation (equation 5):

$\boldsymbol{K}_{i j k l}^{S S}=\boldsymbol{K}_{i j k l}+C_{0} \frac{\boldsymbol{H}_{i j} \boldsymbol{H}_{k l}}{k T}$, with $\boldsymbol{K}_{i j k l}^{S S}$ defined by: $\varepsilon_{i j}=\boldsymbol{K}_{i j k l}^{S S} \sigma_{k l}$

In the presence of stress gradients induced by microstructure or lattice defects in a locally uniform concentration $C_{0}$ of mobile solutes whose associated distortion tensor $\boldsymbol{H}$ is non-zero, the redistribution of solute atoms "in equilibrium with the local stress gradients" will induce a partial relaxation of these gradients. Sofronis and Birnbaum (Sofronis \& H. K. Birnbaum 1995) introduced the concept of "elastic screening of stress centres" to denote this effect. In the case of hydrogen in f.c.c. lattices, we assume that normal lattice interstitial sites are octahedral and that the associated distortion $\Delta v$ is purely diagonal (isotropic dilatation):

$\Delta v=V^{*} / N_{a v}$ 
with $N_{a v}=6.0210^{23}$ and $V^{*}$ the partial molar volume of hydrogen. In cubic crystals, the strain tensor induced by a local hydrogen concentration $C$ is thus diagonal:

$\varepsilon_{i j}^{H}=\frac{\Delta v}{3}\left(C-C_{0}\right) \delta_{i j}$

with $\delta_{i j}$ the Kronecker delta. The distortion tensor $\boldsymbol{H}^{H}$ associated to hydrogen atoms simplifies to:

$\boldsymbol{H}_{i j}^{H}=\frac{\Delta v}{3} \delta_{i j}$

Substituting this expression in that of the chemical potential (6) predicts that hydrostatic tension lowers the chemical potential of solute hydrogen. The first Fick's law becomes:

$\vec{J}=-D_{H}\left(\vec{\nabla} C+\frac{\Delta v}{k T} \cdot C \cdot \vec{\nabla} \frac{\sigma_{k k}}{3}\right)$

The local hydrogen flow is governed by concentration and hydrostatic stress gradients together: it diffuses from compressive zones towards those in tension. From equation (10) and condition (9), one can define "effective" elastic constants at steady state as a function of their value at a given concentration. In the case of an isotropic crystal, the shear modulus, Young's modulus and Poisson's ratio are given by:

$\mu^{H}=\mu$

$E^{H}=\frac{E}{1+\chi \eta^{2} E}$

$v^{H}=\frac{1-\chi \eta^{2} E}{1+\chi \eta^{2} E}$

with $\quad \chi=\frac{c V_{M}}{9 R T} \quad$ and $\quad \eta=\frac{V^{*}}{V_{M}}$

where $V_{M}$ is the molar volume of the host metal, $R$ is the gas constant, and $c$ is the atomic concentration of hydrogen atoms:

$c=\frac{n_{H}}{n_{M}}=\frac{C_{0} V_{M}}{N_{A v}}$ 
In the case of octahedral sites in the f.c.c. lattice, $c$ is also the occupancy of normal interstitial lattice sites. At steady state, given the temperature, molar volume and elastic constants of the host lattice, the modified elastic constants are functions of $c$ only.

\subsection{Hydrogen effects on dislocation properties}

\subsubsection{H-modified self-stress field}

We may now evaluate the stress field around an edge dislocation in the presence of hydrogen. In cylindrical coordinates, using the conventions given in figure 5:

$\sigma_{r r}^{H}=\sigma_{\psi \psi}^{H}=-\frac{\mu b}{2 \pi\left(1-v^{H}\right)} \frac{\sin \psi}{r}$

$\sigma_{r \psi}^{H}=\frac{\mu b}{2 \pi\left(1-v^{H}\right)} \frac{\cos \psi}{r}$

$\sigma_{z z}^{H}=-\frac{\mu b v^{H}}{\pi\left(1-v^{H}\right)} \frac{\sin \psi}{r}$

$\sigma_{r z}^{H}=\sigma_{\psi z}^{H}=0$

To visualise the effects of hydrogen, one may rewrite the radial component of the stress tensor as:

$\sigma_{r r}^{H}=-\frac{\mu b}{2 \pi(1-v)}\left(1-\frac{v-v^{H}}{1-v^{H}}\right) \frac{\sin \psi}{r} \quad$ with $\quad \frac{v-v^{H}}{1-v^{H}}=\frac{1+v}{2\left[1+\frac{9}{2} \cdot \frac{(1-v) R V_{M} \cdot \frac{T}{C}}{E V^{* 2}}\right]}$

From these results, it can be seen that at steady state (i.e.: "in equilibrium with local strain gradients") the self-stress field of an edge dislocation only depends on the temperature, the remote uniform hydrogen concentration $c$, and on materials parameters that are independent on the hydrogen content, including the partial molar volume $V^{*}$. Equations 17-18 are in agreement with Sofronis and correspond to the limit case $t \rightarrow \infty$ of equations 83-85 in (Sofronis 1995). 


\subsubsection{Screening of dislocation pair interactions}

Having a complete description of the hydrogen-modified stress tensor around an edge dislocation, we can now evaluate the consecutive effect on the interaction force between dislocation pairs. Taking two parallel edge dislocations, as depicted on figure 6 , the interaction force between these is given by the Peach and Koehler relation:

$\overrightarrow{F_{u}}=\left(\overrightarrow{b_{2}} \cdot \overline{\bar{\sigma}}\right) \otimes \vec{\xi}$

The radial component $F_{r}$ of this interaction force is then given by:

$F_{r}=b_{2} \sigma_{r \psi} \cos \psi-b_{2} \sigma_{r r} \sin \psi$

Substituting expressions (17.a) and (17.b) in equation (20), one obtains:

$F_{r}=\frac{\mu b_{1} b_{2}}{2 \pi\left(1-v^{H}\right)}\left(\cos ^{2} \psi+\sin ^{2} \psi\right)=\frac{\mu b_{1} b_{2}}{2 \pi\left(1-v^{H}\right)}$

Equation 21 states that, in the presence of a hydrogen solutes distribution in equilibrium with local gradients, the pair interaction force per unit length between the two parallel edge dislocations will be decreased by an amount $1 /\left(1-\nu^{H}\right)$. For a steady-state distribution of solutes, this result is a generalization to non-coplanar configurations of the results obtained numerically by Sofronis (Sofronis \& H. K. Birnbaum 1995) and Chateau (Chateau et al. 2002) on coplanar configurations.

Sofronis proposed a quantitative assessment of the time dependent shielding of pair interactions between two coplanar edge dislocations (Sofronis \& H. K. Birnbaum 1995), based on an iterative finite element analysis scheme to account for hydrogen effect on both dilatation strains and elastic moduli. We published in 2002 a simplified analysis of such a coplanar pair configuration based on a discretisation of the hydrogen concentration field, which only considered dilatation effects and was limited to the steady state distribution (Chateau et al. 2002). In particular, we pointed out that under the condition of steady state, the equilibrium hydrogen distribution and the associated interaction force with dislocations exhibited the same $r^{-1}$ singularity as the glide component of the dislocation pair interaction. To highlight the alteration of pair interactions, we introduced a non-dimensional "screening index", which corresponded to the relative reduction in pair interactions in the presence of an average bulk hydrogen concentration $c$ at temperature $T$ : 
$S(c, T)=\frac{S_{0}}{1+\beta \frac{T}{c}} \quad$ with $\quad \beta=\frac{9(1-v) R V_{M}}{2 E V^{*}}$

In equation $22, S_{0}$ is the non-dimensional maximum amount by which pair interactions can be reduced when $T / c \rightarrow 0$. In the 2002 numerical analysis, which only took dilatation effects into account, we obtained $S_{0}=0.75$, independently of material properties. Taking moduli effects into account in the present analysis (equations 17-18), we obtain:

$S_{0}=(1+v) / 2$

In nickel, $S_{0}=0.64$ and $\beta=2.3310^{-4} \mathrm{~K}^{-1}$.

The fact that, under the assumption of steady state / equilibrium with local gradients, the relative reduction of pair interaction is independent of the dislocation separation distance is a useful result, which we can elaborate on by incorporating the screening index $S(c, T)$ in classical results of dislocation theory to evaluate the effects of hydrogen on elementary dislocation properties.

Making use of the superposition principle in elasticity, this approach also applies to the edge components of dislocations with a mixed character. This property will be used in the analysis of the cross-slip process in section 3.3.2. Finally, it has to be noted that, for non-coplanar configurations, the screening coefficient can be used in the same way as for coplanar pairs, provided only the "glide" component of the interaction force is considered. The "climb" component of the interaction force is not altered in the same way (i.e.: with an $r^{-1}$ singularity) by the solute distribution and the above argument no longer holds (Chateau et al., 2002).

\subsubsection{Line energy and line tension}

As described in $\S 2.1 .1$, in f.c.c. metals, dislocations move by instantaneous jumps between rows of obstacles. As a consequence, line tension has a direct influence on several elementary plasticity mechanisms. Following the classical model of De Wit and Koehler (De Wit \& Koehler 1959), the self-energy per unit length of a dislocation line is defined as the elastic energy embedded in a tube of inner radius $r_{0}$ and outer radius $R$ centred on the dislocation line. It depends on the dislocation character through the angle $\theta$ between the Burgers vector and the dislocation line. The general expression of the line energy $E(\theta)$ is given by: 
$E(\theta)=\frac{\mu b^{2}}{4 \pi K(\theta)} \ln \left(\frac{R}{r_{0}}\right)$ with $K(\theta)=\cos ^{2} \theta+\frac{\sin ^{2} \theta}{1-v}$

where $b$ is the Burgers vector magnitude, $\mu$ the shear modulus, and $v$ the Poisson ratio. The setting of outer and inner cut-off radii $R$ and $r_{0}$ is necessary because the total elastic energy diverges at both extrema. Using H-modified expressions of the dislocation stress field (equation 17), one can express the effect of solute hydrogen on the angular dependence of the line energy $\mathrm{K}(\theta)$ :

$\frac{1}{K(\theta)}=\cos ^{2} \theta+\frac{\sin ^{2} \theta(1-S(c, T))}{1-v}$

where $S(c, T)$ is the screening index defined by equation (22). The exact expression of $\mathrm{K}(\theta)$ is actually more complex than suggested by equations (24-25). Nevertheless, Girardin demonstrated that, for solute concentrations below 0.3, equation (25) was accurate within 1\% (Girardin, 2004).

De Wit and Koehler define the line tension as the first derivative of the self-energy with respect to an incremental increase in line length caused by a small bow-out from an initially straight segment (figure 7):

$\zeta(\theta)=\lim _{\delta L \rightarrow 0}\left(\frac{\delta E(\theta)}{\delta L}\right)$

This definition was used by Friedel in his plasticity textbook (Friedel 1964). Incorporating the screening effect due to hydrogen gives the following expression for the line tension in the De Wit and Koehler / Friedel model:

$\zeta^{d W \& K}=\frac{\mu b^{2}}{4 \pi(1-v)}\left[1-S(T, c)-2(v-S(T, c))+3(v-S(T, c)) \cos ^{2} \theta\right] \ln \left(\frac{R}{r_{0}}\right)$

Jøssang et al. (Jøssang et al. 1965) proposed a more elaborate expression for the line tension, used by Hirth and Lothe (Hirth \& Lothe 1992). In this definition, the variation of elastic interactions between the bowing out segment and the rest of the dislocation line is also taken into account in the energy balance (figure 7). Incorporating hydrogen effects by way of the screening index gives:

$\zeta^{H \& L}=\frac{\mu b^{2}}{4 \pi(1-v)}\left\{\begin{array}{c}\cos ^{2} \theta\left[(1+v-2 S(T, c)) \ln \left(\frac{R^{\prime}}{e \rho}\right)-0.89 v-1.89+2.78 S(T, c)\right] \\ +\sin ^{2} \theta\left[(1-2 v+S(T, c)) \ln \left(\frac{R^{\prime}}{e \rho}\right)+2.78 v-1.89-0.89 S(T, c)\right]\end{array}\right\}$ 
where $R$ ' and $e \rho$ (with $e=2.718$ ) are equivalent to the outer and inner cut-off radii $\mathrm{R}$ and $\mathrm{r}_{0}$ in the Friedel model. The value of the line tension for both models is plotted as a function of the dislocation character for different values of the screening index on figure 8 . The bowing out of an initially straight screw segment creates trailing segments with a non-zero edge component (as for instance the two oblique segments on figure 7). By decreasing the line energy associated to the development of edge components of dislocations, as shown in equations (27) or (28) for $\theta=90^{\circ}$, hydrogen facilitates this bowing out and thus decreases the line tension of screw dislocations.

Most of our modelling developments favour the definition of De Wit \& Koehler / Friedel over that of Jøssang / Hirth \& Lothe, for two reasons:

i. It allows for a closed-form treatment of equations and yields simpler expressions with the same physical meaning.

ii. It is best suited to incorporating these results in Discrete Dislocation Dynamics simulations in future developments, because these simulation codes usually handle line tension effects and elastic pair interactions (including those between different segments of the same dislocation line) in distinct parts.

Nevertheless, the Jøssang / Hirth \& Lothe definition is more accurate for a semi-analytical study of isolated configurations, and the difference between both approaches can be significant (up to $25 \%$ ) for highly curved dislocation segments. This difference vanishes when the curvature decreases. For comparison purposes, we present numerical results for both definitions in the following. Finally, in the absence of hydrogen effects $(S=0)$, equations $27-28$ and onwards reduce to the textbook expressions of dislocation theory (Friedel, 1968; Hirth \& Lothe 1992).

\subsection{Consequences on plasticity mechanisms}

Among others mechanisms, such as the crossing of obstacles rows, line tension controls dislocation multiplication by the Franck-Read mechanism. The above results allow incorporating hydrogen effects in a classical model of this mechanism. Another important mechanism for the development of deformation microstructures, and possibly for damage and fracture processes, is the cross-slip of screw dislocations. A possible effect of hydrogen on the Stacking Fault Energy is pointed out by many authors. The screening of pair interactions will also affect the energy balance for the 
constriction of partial dislocations: we assess this effect and its consequences on the cross-slip probability.

\subsubsection{Multiplication by Franck-Read Sources}

The Franck-Read mechanism for dislocation multiplication is a two-step process:

1. The bowing out of a dislocation segment of finite length pinned at both extremities until a critical unstable configuration is reached. A dislocation loop is then spontaneously injected in the crystal. This translates, via the Peach-Koehler law, into a critical resolved shear stress $\sigma_{\mathrm{c}}$, which depends on the initial segment length and character.

2. The isotropic expansion of the emitted loop which, by decreasing the back-stress on the source like the inverse of the loop radius, will allow the reactivation of emission events.

Both steps depend on the pair interactions and line tension. As such, they are liable to hydrogen effects.

\subsubsection{Critical activation stress}

The starting configuration is that of a finite segment of character $\theta_{0}\left(\theta_{0}=0^{\circ}\right.$ for a screw segment) pinned at both ends on some microstructural defect, and curved under the action of an applied shear stress (figure 9.a) The equilibrium at any point along the line is given by the condition that the Peach-Koehler force and the line tension should be opposed (figure 9.b):

$\sigma b \vec{N}=-\kappa \zeta(\theta) \vec{N}$

with $\kappa$ the segment curvature and $\vec{N}$ its normal in the glide plane. This equation corresponds to the following partial differential equation:

$\sigma b \vec{N}=-\frac{d}{d s}\left[E(\theta) \vec{T}+\frac{d E(\theta)}{d \theta} \vec{N}\right]$

Solving this system for $\sigma$ is fairly simple using the De Wit \& Koehler line tension definition, and has to be carried out by numerically with the Jøssang definition. The result is a critical value of the 
resolved shear stress $\sigma_{c}$, which can be expressed like in equation 31, where $\sigma_{c}^{*}$ is the nondimensional part that depends on the character $\theta$ and the screening index $S(c, T)$ :

$\sigma_{c}(\theta)=\frac{\mu b}{L} \sigma_{c}^{*}(\theta, S) \ln \left(\frac{\mathrm{R}}{\mathrm{r}_{0}}\right)$

The results are plotted in figure 10 for both line tension definitions. In the absence of hydrogen $(\mathrm{S}=0)$, screw dislocations are more rigid than edge segments, as pointed out by Ashby (Ashby 1966). Following line tension calculations (equations 27-28), increasing the screening index tends to decrease the stiffness of screw segments. In this case, the critical activation stress is significantly decreased for segments that are predominantly screw. Similarly, pure edge segments remain unaffected in the De Wit \& Koehler approach, whereas they are only slightly influenced by $S(T, c)$ in the Jøssang model.

\subsubsection{Isotropic loop expansion}

Once emitted, a dislocation loop will exert a back-stress on the segment from which it was emitted and impede the repeated operation of the source. Isotropic loop expansion under an applied shear stress allows to decrease this back-stress and to reactivate the emission process. Hydrogen segregation on the edge components of the loop will both decrease the magnitude of the back-stress and the line energy of the total loop, which opposes to its isotropic extension. Following Hirth \& Lothe (Hirth \& Lothe 1992) we approach the shape of a circular loop of radius L by that of a hexagon of side L (see Figure 11), which is accurate within 1\% for loops of radius 100b or above. The total energy of such a loop configuration is the sum of the line energy $W_{\text {self }}$ and the elastic interaction energy between segments $W_{\text {int }}$. Using equations 22 and 25, we obtain:

$W_{\text {hex.loop }}^{H}=W_{\text {self }}^{H}+W_{\text {int }}^{H}=\frac{3 \mu b^{2} L}{4 \pi(1-v)} \cdot[2-v-S(T, c)] \cdot\left[0.16+\ln \left(\frac{L}{r}\right)\right]$

The force for isotropic loop expansion is given by the derivative of this energy with respect to the loop radius L:

$F=\frac{\partial W}{\partial L}$ 
In the same way as with the interaction force across dislocation pairs, we express the Hydrogenaffected value of this expansion force by normalizing it with its value in the hydrogen-free crystal. We obtain the dimensionless ratio:

$\frac{F^{H}}{F} \approx 1-0.58 S(T, c)$

Solid solution hydrogen tends to favour dislocation multiplication by decreasing both the critical activation stress of Franck-Read sources and the force for isotropic loop expansion. In a deforming crystal, this is expected to assist flow localization, by favouring the activity of existing sources against the activation of fresh ones.

\subsubsection{Cross-slip probability of screw dislocations}

The cross-slip of screw dislocations is one of the most important elementary plasticity mechanisms in f.c.c. crystals. It affects directly the strain hardening via the self-hardening of individual slip systems, the development of deformation microstructures, and the build-up of internal stresses by the formation of dislocation pile-ups against interfaces or grain boundaries. Scanning Electron Microscopy (SEM) observations of slip traces at the surface of deformed samples led to numerous reported observations of an increase of slip planarity and slip band localization in hydrogencontaining samples (Ulmer \& Altstetter 1991). Aubert et al. (Aubert et al. 2010) recently conducted a quantitative analysis of this localization by means Atomic Force Microscopy (AFM) combined with a Crystal Plasticity Finite Element Analysis (CP-FEA) of the slip activity necessary to build the measured surface steps. In a 316L strained by $6 \%$ in tension, they observed that, for the same amount of deformation, the intense slip bands in hydrogen-containing samples (40 wt.ppm) were twice fewer and corresponded to average slip steps 50 to $60 \%$ higher than in hydrogen-free samples. This increased slip planarity is commonly attributed to a decrease of the alloy's Stacking Fault Energy (SFE) in the presence of hydrogen. Indeed, such a decrease can be predicted from first principles calculations. The question however remains on whether it is quantitatively sufficient to account for the observed effects. Ferreira et al. (P J Ferreira et al. 1996) published unique in-situ observations of H-effect on the width of extended triple nodes in an austenitic stainless steel, obtained in the environmental TEM at the University of Illinois. Using a classical model to interpret 
these observations, they concluded to a $20 \%$ decrease of the SFE under a hydrogen partial pressure of $20 \mathrm{~Pa}$, combined with a Pd coating of thin foils. In the absence of other effects, a $20 \%$ decrease of the SFE alone is indeed insufficient to account for the observed localization effects. A more detailed analysis of the cross-slip process and the associated energy barrier is necessary.

\subsubsection{H-effects on the dissociated configuration}

Figure 12 represents the dislocation configuration involved in the cross-slip process in an f.c.c. crystal: a perfect dislocation with a Burgers vector $1 / 2<110\rangle$ is dissociated in two Shockley partials of Burger's vector $1 / 6<112>$ that bound a stacking fault ribbon. The Burgers vectors of the partials are repulsive with respect to each other, with a pair interaction force scaling like the inverse of the separation distance $\mathrm{d}_{0}$. The energy cost of the stacking fault is proportional to its surface. Assuming parallel straight dislocation segments, it depends linearly on the separation distance $\mathrm{d}_{0}$. This yields an attractive configurational force of constant magnitude, equal to $\Gamma$, the Stacking Fault Energy (SFE). The balance between these two forces defines the equilibrium configuration:

$\Gamma=\frac{F_{R}}{L}=-\frac{\partial\left(\frac{W_{12}}{L}\right)}{\partial R}=\frac{\mu}{2 \pi d_{0}}\left[\left(\vec{b}_{1} \cdot \vec{\xi}_{1}\right)\left(\vec{b}_{2} \cdot \vec{\xi}_{2}\right)+\frac{\left(\vec{b}_{1} \otimes \vec{\xi}_{1}\right)\left(\vec{b}_{2} \otimes \vec{\xi}_{2}\right)}{1-v}\right]$

$W_{12} / L$ is the work per unit of line length $L$ of the interaction force between the two partials associated to a variation of their separation distance $R$, and $F_{R}$ is the associated configurational force. Using equation (21) for the edge components of the partial dislocations, we can express the equilibrium separation distance in the presence of hydrogen:

$d_{0}^{H}=\frac{\mu b_{p}^{2}}{8 \pi \Gamma} \frac{((2-S(T, c))-(v-S(T, c))(1+2 \cos 2 \theta))}{1-v}$

For $\theta<\pi / 6$, the dominating contribution is the repulsion between the screw components of the partials, but edge components are attractive and $\mathrm{H}$-induced screening decreases this attractive interaction. The net effect is thus an increase of the repulsion between partials and Hydrogen solutes, which participates in the widening of the stacking fault ribbon. For cross-slip, screw dislocations are involved $(\theta=0)$ and equation (38) reduces to:

$\left.d_{0}^{H}\right|_{\text {screw }}=\frac{\mu b_{p}^{2}}{8 \pi \Gamma} \frac{2-3 v+S(T, c)}{1-v}$ 


\subsubsection{H-effects on the recombination work and cross-slip probability}

Cross-slipping of screw dislocations is a thermally activated process for which the energy barrier corresponds to the work for constricting and recombining Shockley partials into a locally perfect screw segment before it deviates in its secondary plane. Since H-induced screening of pair interactions decreases the repulsive component between partials of a dissociated screw dislocation (equation 38), it participates in the increase of this recombination work in addition to a possible decrease of the SFE. In our analysis of 2002 (Chateau et al.2002), we compared the magnitude of both contributions for the same "apparent" effect on the separation distance and concluded that the contribution of the screening effect dominated: integrating this effect over the constriction path amounted to integrating a contribution singular with respect to the separation distance ( $\alpha 1 / \mathrm{d})$, against a constant one in the case of the effect on the SFE. However, this 2D analysis implicitly assumed that the constriction operated on rigid parallel segments. After Bonneville \& Escaig (Bonneville \& Escaig 1979) and more recent contributions, we know that the cross-slip process is fundamentally three-dimensional, with a constriction taking place locally along the dislocation line and extending sidewise, while a new dissociated segment bows out in the cross-slip plane (Bonneville \& Escaig 1979). In this respect, the 2002 analysis, based on rigid parallel segments, gave an upper bound for the contribution of $\mathrm{H}$-induced screening on the cross-slip probability. Several models and experimental studies are available to estimate the dependence of the constriction work with the equilibrium separation distance. They were reviewed and compiled by Püschl (Püschl \& Schoeck 1993; Püschl 2002), who derived the following semi-empirical relation between the two quantities:

$\frac{E_{c}}{K_{S} b^{3}} \approx 1.2 \times 10^{-2} \frac{d}{b} \ln \left(2 \frac{d}{b}\right)$ for a screw dislocation in isotropic elasticity

with $d$ the separation distance, $E_{c}$ the constriction work per unit dislocation length, and $K_{s}$ the prelogarithmic constant associated to the cut-off radius (equation 24). Substituting the expression of the equilibrium dissociation distance $d_{0}{ }^{H}$ as a function of the hydrogen content (equation 39) gives a relative increase of the constriction work that depends almost linearly on the screening index $S(T, c)$ (plotted for a constant value of $\Gamma$ on Figure 13). Combined with the effect of hydrogen on the SFE, both effects add-up to decrease significantly the cross-slip probability, even for relatively low hydrogen contents and/or in alloys with an intermediate value of the SFE. In addition, in situ 
observations by Ferreira et al. (Ferreira et al., 1998) during deformation of aluminium foils in the environmental TEM under $\mathrm{H}_{2}$ pressure showed that the cross-slipping of dislocations was also impeded by the stabilization of edge segments at the expense of screw parts in curved loops. This effect can be predicted from the line energy / line tension calculations presented in section 3.2.3. All these contributions are quantitatively insufficient to account on their own for the observed effects of hydrogen on slip planarity, but they all go in the same direction: a decrease of the cross-slip probability in deformed crystals containing hydrogen. A quantitative model integrating all these contributions remains to be developed.

\subsection{Summary of modelling results}

We defined a non-dimensional quantity $\mathrm{S}(\mathrm{T}, \mathrm{c})$ that measures the relative amount by which the Peach-Koehler force between two parallel edge dislocations is reduced in the presence of a solute distribution in equilibrium with local stress gradients (equation 22). This screening index was used to derive hydrogen-modified expressions for the line energy (equations 24-25), line tension (equations 27-28) and pair interactions between dislocations (equations 22-23). These expressions can be used to evaluate the effects of mobile hydrogen solutes on elementary plasticity mechanisms, such as the onset of a Franck-read source (equation 31), the isotropic expansion of a hexagonal loop (equations 32-34), or the separation distance between partials (equations 38-39), which controls the thermal activation for cross-slip. These modelling results are now compared to the mechanical response of $\mathrm{H}$-charged single crystals.

\section{Experiments on f.c.c. single crystals oriented for single glide}

In this section, we report deformation experiments on model materials that illustrate the modelling results presented the previous section. As stated in the introduction of this chapter, in f.c.c. crystals, long range pair interactions have a limited and indirect influence on the flow properties of these alloys. Modifying these by ways of solute hydrogen introduction may have multiple effects on the net flow stress, and only in rare occasions can these be interpreted as resulting directly from $\mathrm{H}$ effects on dislocation pair interactions. The cyclic plasticity of single crystals oriented for single 
glide is one of these: we examine this situation first. Interpreting a tensile curve in terms of elementary dislocation properties is more indirect, but can be done using single crystals and Transmission Electron Microscopy (TEM). We investigate the three stages of work-hardening in single crystals oriented for "single glide".

The materials preparation, including hydrogen charging, is similar to the procedure described in section 2.2.1. Smooth specimens with a 4-mm wide square section and a 10-mm gauge length were cut in pure Ni single crystals along the [153] direction, corresponding to a "single slip" orientation (Schmid factor 0.49). Non-charged specimens were exposed to a neutral atmosphere at the same temperature and for the same time as $\mathrm{H}$-charged ones (typically 48 hours at $450^{\circ} \mathrm{C}$ ), to account for possible recovery effects during hydrogen charging in autoclave. They were tested in symmetric tension-compression and monotonous tension.

\subsection{Cyclic plasticity}

Cyclic plasticity tests on hydrogenated nickel single crystals are thoroughly described and analysed in (Magnin et al. 2001). We recall the main results and conclusions. Tension-compression tests were conducted at a varying applied plastic strain amplitude $\Delta \varepsilon_{\mathrm{p}} / 2$ for a plastic strain rate of $10^{-3}$ $\mathrm{s}^{-1}$. Figure 14 displays the cyclic stress-strain curves for Nickel and hydrogenated Nickel. These curves correspond to the saturated value of the cyclic flow stress as a function of the applied plastic strain amplitude. They display 3 characteristic regions (Mughrabi 2001; Mughrabi 1979). The first one (small amplitudes) corresponds to the matrix-vein dislocation structure. In this region, the cyclic flow stress increases with the applied strain amplitude. The second one is a plateau region, typical of the Persistent Slip Band (PSB) structure, in which the cyclic strain is accommodated by back-and-forth movements of screw dislocations within stable channels delimited by PSB walls of edge character. At larger strain amplitudes, the PSB structure colonizes the whole specimen gauge length and eventually collapses (Laird et al. 1986; Essmann \& Mughrabi 1979).

The onset of the PSB regime is known to be controlled by the cross-slip probability. The comparison between hydrogen-free and hydrogen-containing specimens shows that, in this respect, the cyclic stress-strain curve is shifted towards the right-hand side with the introduction of hydrogen. This result is a direct macroscopic evidence of the effect of solute hydrogen on the 
decrease of the cross-slip probability. This is confirmed by the shape of the hysteresis loops (saturated stress-strain cycles at given values of the shear amplitude). We know from Essmann and Mughrabi (Essmann \& Mughrabi 1979; Mughrabi 1979) that, in the PSB region, these hysteresis loops tend to be more rectangular with increasing plastic strain amplitudes. At a given plastic shear amplitude; uncharged $\mathrm{Ni}$ curves are indeed slightly more rectangular than for hydrogen-containing samples (Figure 15). It confirms that the shift observed at the onset of the PSB regime for hydrogenated samples is kept retained across the whole plateau region. This shift is caused by a decrease of the cross-slip probability. One may also notice that the cyclic stress in the plateau region in lowered in the presence of hydrogen. This value is related to the annihilation by double cross-slip of screw segments moving in the channels, which has to be compensated for by the emission of new edge segments from the walls aligned along the edge direction (Essmann \& Mughrabi 1979; Mughrabi 1979). From the modelling results, we can predict that both phenomena are affected by the presence of hydrogen solutes, thus participating in the observed cyclic softening.

\subsection{Monotonous tension}

\subsubsection{Tensile behaviour}

In this section, we present experimental results in tension of non-charged and hydrogen-charged nickel single crystals oriented for single slip. Hydrogen charging of these samples gives a 1600 at. ppm hydrogen concentration. Stress strain curves for nickel and nickel-hydrogen at a plastic strain rate of $10^{-2} \mathrm{~s}^{-1}$ are shown in Figure 16.b, along with a schematic representation of the three stages of work hardening in f.c.c. single crystals oriented for single glide (Figure 16.a). Comparing the stressstrain curves of $\mathrm{Ni}$ and $\mathrm{Ni}-\mathrm{H}$ crystals, one can notice that the overall behaviour cannot be rationalized simply in terms of "softening" or "hardening" induced by solute hydrogen: different contributions add up with different relative weights, depending on the strain level. Decomposing the stressstrain curves in successive stages, where different plasticity mechanisms prevail, allows identifying the following features related to hydrogen charging:

- Hydrogen causes a noticeable increase of the magnitude of $\tau_{\mathrm{I}}\left(\sim 0.0510^{-3} \mu\right)$, the critical stress for the onset of stage I deformation. At room temperature, mobile dislocations are continuously 
aged during straining by mobile hydrogen atoms. This hardening contribution induced by hydrogen is attributed to the viscous drag of solute atmospheres.

- Hydrogen delays the emergence of stage II (linear hardening, see figure 16.a). In stage I ("easy glide"), a unique slip system is active. Plastic deformation during this stage is accommodated by the activation of Frank-Read sources. Few obstacles impede the glide of emitted dislocation loops, resulting in a very low work hardening rate. According to the results presented in the previous section, both multiplication and planar glide are expected to be favoured by solute hydrogen. The termination of stage I and transition to stage II is directly related to the selfhardening of the primary slip system, which is in turn controlled by the formation of collinear junctions (Madec et al. 2003). These junctions may have a non-zero of edge character, in which case the modelling results allow predicting their weakening in the presence of solute hydrogen, through the screening of pair interactions. This contribution is a direct macroscopic manifestation of the "hydrogen induced softening" predicted by Birnbaum and co-workers at the scale of dislocation.

- In stage II, the introduction of hydrogen in nickel results in a significant increase of the work hardening. This stage of rapid linear hardening is characterised by the activation of secondary slip systems and the work hardening is governed by the interaction between dislocations from the first activated system and those from secondary slip systems. The flow stress is essentially controlled by the dislocation mean free path, which will be investigated by transmission electron microscopy.

These results provide more experimental evidences of hydrogen effects on elementary plasticity mechanisms. The observed hydrogen effects on stage I (increase of $\tau_{\mathrm{I}}$ due to solute drag and extension of the planar glide stage) are consistent with (Yagodzinskyy et al. 2010) on 18Cr-16Ni-10Mn austenitic stainless steel single crystals with a higher $\mathrm{H}$ content.

\subsubsection{TEM observations}

Dislocation structures were compared between Ni and Ni-H by Transmission Electron Microscopy (TEM). TEM foils were sampled in deformed $\mathrm{Ni}$ and $\mathrm{Ni}-\mathrm{H}$ single crystals after 0.75 plastic shear strain, i.e.: where the two curves intersect on figure 16.b. At this strain level, both crystals have the same flow stress, but different strain hardening rates. It can be seen from figures 17-18 that the 
increased strain hardening rate in $\mathrm{Ni}-\mathrm{H}$ stems from the higher dislocation density in this specimen. Moreover, the dislocation structures themselves display very different features. The nickel single crystal is well in the stage III regime, with the formation of equiaxed dislocation cells (figure 17). On the contrary, the hydrogenated sample displays a planar structure of dislocation walls, typical of alloys with a low SFE, where the low probability of cross-slip prevents the onset of dynamic recovery and the formation of dislocation cells (figure 18). This demonstrates the effects of solute hydrogen on the cross-slip mechanism, and its indirect consequences on the strain hardening rate, via a decrease of the dislocation mean free path.

\section{Review of main results and conclusions}

In this chapter, we first investigated the dynamic interactions between mobile dislocations and mobile hydrogen solutes. In hydrogenated nickel and nickel-chromium at ordinary strain rates, the mobilitv of $\mathrm{H}$ solutes and that of dislocations around 5-7 \% of plastic strain are of the same order in the temperature range $-120^{\circ}$ to $-60^{\circ} \mathrm{C}$. Static Strain Ageing experiments at lower temperatures allow the measurement of the binding between temporarily arrested dislocations and their $\mathrm{H}$ atmosphere as a function of the Hydrogen content. This pinning stress is of the order of 15 to $20 \mathrm{MPa}$ for Hydrogen concentrations between 1500 and 2000 at.ppm, and is an increasing function of this concentration. At room temperature during monotonous deformation, dislocations are thus continuously aged to saturation, giving a constant positive contribution to the flow stress of the same amount ("solute drag" force). This contribution is expected to be present in all f.c.c. alloys containing mobile $\mathrm{H}$ solutes, along with others that may be of both signs, as shown in section 4 .

In the section on modelling at the scale of discrete dislocations, we first recalled the theoretical framework of "linearized hydrogen elasticity" proposed by Sofronis and Birnbaum and derived a closed form expression for the self-stress field of a dislocation in the presence of solute hydrogen under the assumption of steady state / equilibrium with local gradients of solute distribution. We pointed out a property of the resulting equilibrium hydrogen distribution which allowed incorporating the effects of mobile hydrogen atoms in a simple scalar quantity, function of the hydrogen concentration, temperature and elastic constants of the host lattice: the "screening index". 
$\mathrm{S}(\mathrm{T}, \mathrm{c})$ was then introduced in classical expressions of the line energy and line tension, enabling an estimate of $\mathrm{H}$ effects on plasticity mechanisms involving simple configurations, such as multiplication and cross-slip.

Deformation experiments on single crystals, combined to TEM investigations allowed the confrontation of these modelling results to experiments. The first effect is an increase of the critical stress for the onset of plasticity, by an amount equivalent to the "solute drag" force measured in Static Strain ageing. Both cyclic and monotonous deformation of single crystals oriented for single glide displays distinct stages. The transition between these successive stages is controlled by the cross-slip probability. Hydrogen addition to Nickel crystals has the effect of delaying these transitions, thus highlighting the decrease of cross-slip probability as the most direct effect of hydrogen on deformation. This has further consequences on the hardening rate in Stage II (tensile) deformation, via the development of a dense array of planar dislocation walls, instead of a cellular microstructure. Finally, the cyclic flow stress in the PSB regime is controlled by a balance between multiplication by the bowing of edge segments out of PSB walls and the annihilation of gliding screws by double cross-slip within the channels. Both effects can be rationalised within the modelling framework presented in section 3.

Finally, the strain ageing and tensile experiments on single crystals highlighted various direct or indirect contributions of hydrogen to the flow stress, with both signs. The net "softening" or "hardening" character of solute hydrogen is then a matter of weighting these different contributions as a function of the deformation (the "Ni" and "Ni-H" curves on Figure 16.b cross each other twice) and alloy metallurgy. Indeed, an important aspect pointed out by Sofronis et al. (Sofronis et al., 1993) and ignored in the present experimental study on "pure" metals is the important role of the impurity content, through the screening effect of hydrogen on the interactions between dislocations and solid solution hardening atoms such as carbon. This effect is expected to be of prior importance in engineering structural alloys.

\section{Directions for further developments}


The formalism of "hydrogen-elasticity" can be introduced in classical expressions of dislocation properties, provided they pertain to the linear elastic theory of discrete dislocation. Properties which derive from the core structure of crystal defects such as the saddle point configuration of constricted partials during cross-slip or the trapping of hydrogen solutes in complex grain boundary structures have to be handled by atomistic approaches before one can derive simple models to be used at the scale of discrete defects. Similarly, for high levels of alloying such as encountered in engineering grades of Ni-based alloys and austenitic stainless steels, other contributions like short-range order effects are liable to be affected by solid solution hydrogen. A quantitative approach of these should combine atomic scale simulations and linear elasticity. At a larger scale, Crystal Plasticity Finite Elements (CP-FEM) simulations are an efficient way of predicting the response of polycrystalline aggregates from single crystal behaviour. CP-FEM models are essentially made of a viscoelastoplastic law for isolated slip systems and an interaction matrix describing the interactions between individual systems (Barbe 2001). In f.c.c. crystals however, collective effects play an important part (Devincre et al. 2008). 3-Dimensional Discrete Dislocation Dynamics (DDD) simulations may be used to identify the coefficients of the interaction matrix (Queyreau et al. 2009). The formalism presented in this chapter can be used to introduce hydrogen effects in DDD simulations and derive hydrogen-modified rules for the interactions between slip systems.

Experimental data on model systems remains necessary to sort out the numerous possible effects of solid solution hydrogen on deformation. The experimental methodology introduced in this chapter, although relevant to validating the type of modelling presented here, has limitations related to the range of attainable solid solution concentration. Higher levels of supersaturation are necessary to approach conditions related to hydrogen-induced fracture in Stress Corrosion Cracking for instance. Physically-based modelling of brittle fracture in engineering alloys require altogether the derivation of a theoretical decohesion criterion and modelling the dynamics of crack-tip plasticity (Roberts, 1999). Modelling hydrogen effects on decohesion requires the development of specific atomic scale methods (Tanguy, 2007). The present analysis provides ways of introducing Hydrogen effects on dislocation interactions in Discrete Dislocation Dynamics simulations of crack-tip plasticity. However, adsorbed Hydrogen is also liable to affect the dislocation nucleation criterion at a loaded crack-tip (Tanguy et al., 2008), a quantity which is difficult to evaluate experimentally. Recent developments in the testing of micrometer-sized samples in Hydrogenating environments may 
provide a fairly direct access to H-effects on dislocation multiplication (Barnoush et al. 2010; Barnoush \& Vehoff 2010).

Finally, the present contribution focuses on f.c.c. metals and alloys. Although the framework of linear elasticity presented here does not assume any specific crystallography, extending this approach to other metal systems may require alternative experimental and modelling efforts because other contributions may prevail. The case of hydride-forming systems is a well-known example (Gahr et al. 1980). Another important area of interest is b.c.c. iron and iron-based alloys. In these alloys, the hydrogen solubility is much lower than in their f.c.c. counterparts. The contribution of elastic interaction effects should be somewhat limited. On the other hand, complex collective effects are less important in these materials and "one-dislocation models" often provide relevant predictions. Nano-indentation experiments demonstrated important effects of hydrogen on dislocation multiplication, together with a possibly important hardening contribution, even in the presence of small hydrogen quantities (Katz 2001). The low temperature mobility of dislocations by the double kink mechanism is a key parameter for predicting the brittle to ductile transition in pure iron (Tanaka et al., 2008 ). In terms of plasticity mechanisms, investigating the effects of solute hydrogen on the transition temperature for the mobility of screw dislocations should be of particular interest in these systems.

Acknowledgement: The author gratefully acknowledges the contribution of Pr. X. Feaugas of Laboratoire des Sciences de 1'Ingénieur pour l'Environnement (LaSIE) FRE-CNRS 3474, La Rochelle, France, for performing the TEM observations and for his constant interest in this work.

\section{References}

Ashby, M., 1966. Results and consequences of a recalculation of the frank-read and the orowan stress. Acta Metallurgica, 14(5), pp.679-681. 
Aubert, I., Olive, J.-M. \& Saintier, N., 2010. The effect of internal hydrogen on surface slip localisation on polycrystalline AISI 316L stainless steel. Materials Science and Engineering: A, 527(21-22), pp.5858-5866.

Bastien, P. and Azou, P., 1951, Influence of the amplitude and of the speed of plastic deformation of the segregation of hydrogen in iron and steels. Comptes Rendus Hebdomadaires des Seances de l'Academie des Sciences, 232, p. 69

Barbe, F., 2001. Intergranular and intragranular behavior of polycrystalline aggregates. Part 1: F.E. model. International Journal of Plasticity, 17(4), pp.513-536.

Barnoush, A. \& Vehoff, H., 2010. Recent developments in the study of hydrogen embrittlement: Hydrogen effect on dislocation nucleation. Acta Materialia.

Barnoush, A., Zamanzade, M. \& Vehoff, H., 2010. Direct observation of hydrogen-enhanced plasticity in super duplex stainless steel by means of in situ electrochemical methods. Scripta Materialia, 62(5), pp.242-245.

Birnbaum, H. K., 1994, Hydrogen effects on deformation-relation between dislocation behavior and the macroscopic stress-strain behavior. Scripta Metallurgica et Materialia, 31, p. 149

Birnbaum, H. \& Sofronis, P., 1994. Hydrogen-enhanced localized plasticity—a mechanism for hydrogen-related fracture. Materials Science and Engineering: A, 176(1-2), pp.191-202.

Bonneville, J. \& Escaig, B., 1979. Cross-slipping process and the stress-orientation dependence in pure copper. Acta Metallurgica, 27(9), pp.1477-1486.

Brass, A., 1996. Measurements of deuterium and tritium concentration enhancement at the crack tip of high strength steels. Corrosion Science, 38(4), p.569-585.

Chateau, J.P., Delafosse, D. \& Magnin, T., 2002. Numerical simulations of hydrogen - dislocations interactions in fcc stainless steels - Part I : hydrogen - dislocations interactions in bulk crystals. Acta Materialia, 50(6), pp.1507-1522. 
Chene, J. \& Brass, A.M., 1990. In R. Oltra \& D. Desjardin, eds. Corrosion Sous Contrainte Phénoménologie et Mécanismes. Les Ulis, France: Les Editions de Physique, pp. 159-210.

Chene, J. \& Brass, A. M., 1999. Hydrogen transport by mobile dislocations in nickel base superalloy single crystals. Scripta Materialia, 40(5), p.537-542.

Chene, J. et al., 2007. Role of microstructure and heat treatments on the desorption kinetics of tritium from austenitic stainless steels. Journal of Nuclear Materials, 360(2), p.177-185.

Chihab, K. et al., 1987. The kinetics of the Portevin-Le Chatelier bands in an Al-5at\% Mg alloy. Scripta Metallurgica, 21(2), pp.203-208.

Combette, P. \& Grilhe, J., 1970. Portevin-Le Chatelier effect and discontinuity at yield point in hydrogenated nickel. Memoires Scientifiques de la Revue de Metallurgie, 67(7-8), pp.491-505.

De Wit, G. \& Koehler, J.S., 1959. Interaction of dislocations with an applied stress in anisotropic crystals. Physical Review, 116, pp.1113-1120.

De, A., 1999. Static strain aging behavior of ultra low carbon bake hardening steel. Scripta Materialia, 41(8), pp.831-837.

Devincre, B., Hoc, T. \& Kubin, L.P., 2008. Dislocation Mean Free Paths and Strain Hardening of Crystals. Science, 320, pp.1745-1748.

Donovan, J. E., 1976, Sorption of tritium by nickel during plastic deformation. Metallurgical Transactions A, 7(1), pp.145-149

Essmann, U. \& Mughrabi, H., 1979. Annihilation of dislocations during tensile and cyclic deformation and limits of dislocation densities. Philosophical Magazine a-Physics of Condensed Matter Structure Defects and Mechanical Properties, 40(6), pp.731-756.

Ferreira, P J, Robertson, I M \& Birnbaum, H K, 1996. Influence of hydrogen on the stacking-fault energy of an austenitic stainless steel. In Ferro, MA, ed. Intergranular and interphase boundaries in materials, PT 1. Transtech Publications Ltd, pp. 93-96. 
Ferreira, P.J., Robertson, I.M. \& Birnbaum, H.K., 1998. Hydrogen effects on the interactions between dislocations. Acta Materialia, 46(5), pp.1749-1755.

Ferreira, P.J., Robertson, I.M. \& Birnbaum, H.K., 1999, Hydrogen effects on the character of dislocations in high-purity aluminium, Acta Materialia, 47(10), pp. 2991-2998

Friedel, J., 1964. Dislocations, Oxford: Pergamon Press.

Gahr, S., Makenas, B. \& Birnbaum, H., 1980. Fracture of niobium hydrid. Acta Metallurgica, 28(9), pp.1207-1213.

Girardin, G., 2004. Interactions hydrogène - plasticité dans le nickel et ses alliages. Saint-Etienne: Ecole Nationale supérieure des Mines et Université Jean Monet.

Girardin,G. \& Delafosse, D., 2004, Measurement of the saturated dislocation pinning force in hydrogenated nickel and nickel base alloys, Scripta Materialia, 51, pp. 1177-1181

Hirth, J.P. \& Lothe, J., 1992. Theory of dislocations 2nd ed., Malabar FL: Krieger Pub. Co.

Jøssang, T., Lothe, J. \& Skylstad, K., 1965. Explicit expressions for the energy of dislocation configuration made up of piecewise straight segments. Acta Metallurgica, 13, pp.271-278.

Katz, Y., 2001. Nanomechanical probes as new approaches to hydrogen/deformation interaction studies. Engineering Fracture Mechanics, 68(6), pp.619-646.

Kocks, U. F. \& Mecking, H., 2003, Physics and phenomenology of strain hardening : the FCC case, Progress in materials science, 48(3), pp.171-173

Kubin, L.P., Estrin, Y. \& Perrier, C., 1992. On static strain ageing. Acta Metallurgica et Materialia, 40(5), pp.1037-1044.

Kubin, L.P., Chihab, K. \& Estrin, Y., 1988. The rate dependence of the portevin-Le chatelier effect. Acta Metallurgica, 36(10), pp.2707-2718. 
Kubin, L.P. \& Estrin, Y., 1990. Evolution of dislocation densities and the critical conditions for the Portevin-Le Châtelier effect. Acta Metallurgica et Materialia, 38(5), pp.697-708.

Laird, C., Charlsey, P. \& Mughrabi, H., 1986. Low-energy dislocation structures produced by cyclic deformation. Materials Science and Engineering A, 81(1-2), pp.433-450.

Larche, F. \& Cahn, J.W., 1985. Overview no. 41 The interactions of composition and stress in crystalline solids. Acta Metallurgica, 33(3), pp.331-357.

Madec, R. et al., 2003. The Role of Collinear Interaction in Dislocation-Induced Hardening. Science, 301(5641), pp.1879-1882.

Magnin, T. et al., 2001. Cyclic plastic deformation behaviour of Ni single crystals oriented for single slip as a function of hydrogen content. Materials Science and Engineering A, 314, pp.7 11.

Mccormick, P., 1988. Theory of flow localisation due to dynamic strain ageing. Acta Metallurgica, 36(12), pp.3061-3067.

Mughrabi, H., 2001. Cyclic strain localization in fatigued metals. In Bouchaud, E, ed. NATO Science Series II: Mathematics, Physics and Chemistry : Physical Aspects of Fracture, 32, pp. 271-281.

Mughrabi, H., 1979. Plateaus in the cyclic stress-strain curves of single -crystalline and polycrystalline metals. Scripta Metallurgica, 13(6), pp.479-484.

Puschl, W. \& Schoeck, G., 1993. Calculation of cross-slip parameters in f.c.c. crystals. Materials Science and Engineering: A, 164(1-2), pp.286-289.

Püschl, 2002. Models for dislocation cross-slip in close-packed crystal structures: a critical review. Progress in Materials Science, 47, pp.415-461.

Queyreau, S., Monnet, G. \& Devincre, B., 2009. Slip systems interactions in $\alpha$-iron determined by dislocation dynamics simulations. International Journal of Plasticity, 25(2), pp.361-377. 
Roberts, S.G., 1999. Modelling Brittle-Ductile Transitions. In Multiscale Phenomena in Plasticity : from Experiments to Phenomenology, Modelling \& Materials Engineering.

Samuel, K.G., Mannan, S.L, Rodriguez, P., 1988, Serrated yielding in AISI 316 stainless steel, Acta Metallurgica. 36(8), p. 2323.

Sofronis, P., 1995. The influence of mobility of dissolved hydrogen on the elastic response of a metal. Journal of the Mechanics and Physics of Solids, 43(9), pp.1385-1407.

Sofronis, P. \& Birnbaum, H. K., 1995. Mechanics of the hydrogen-dislocation-impurity interactions--I. Increasing shear modulus. Journal of the Mechanics and Physics of Solids, 43(1), pp.49-90.

Sofronis, P. \& Robertson, I.M., 2002. Transmission electron microscopy observations and micromechanical/continuum models for the effect of hydrogen on the mechanical behaviour of metals. Philosophical Magazine a-Physics of Condensed Matter Structure Defects and Mechanical Properties, 82(17-18), pp.3405-3413.

Tanaka, M., Tarleton, E. \& Roberts, S.G., 2008. The brittle-ductile transition in single-crystal iron. Acta Materialia, 56(18), pp.5123-5129.

Tanguy, D., 2007, Constrained molecular dynamics for quantifying intrinsic ductility versus brittleness, Physical Review B, 76(14)

Tanguy, D., Razafindrazaka, M. \& Delafosse, D., 2008. Multiscale simulation of crack tip shielding by a dislocation. Acta Materialia, 56, pp.2441-2449.

Ulmer, D. \& Altstetter, C., 1991. Hydrogen-induced strain localization and failure of austenitic stainless steels at high hydrogen concentrations. Acta Metallurgica et Materialia, 39(6), pp.1237-1248.

Varadhan, S., Beaudoin, A.J. \& Fressengeas, C., 2009. Lattice incompatibility and strain-aging in single crystals. Journal of the Mechanics and Physics of Solids, 57(10), pp.1733-1748. 
Wilcox, B.A. \& Smith, G.C., 1964. The Portevin-Le Chatelier effect in hydrogen charged nickel. Acta Metallurgica, 12, pp.371-376.

Windle, A. H. and Smith, G. C., 1968. Effect of hydrogen on plastic deformation of nickel single crystals. Metal Science Journal, 2, 187.

Yagodzinskyy, Y. et al., 2010. Effect of hydrogen on plastic strain localization in single crystals of austenitic stainless steel. Scripta Materialia, 62(3), pp.155-158. 


\section{Figures}

1

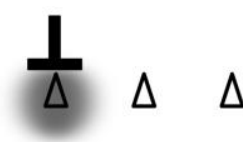

3

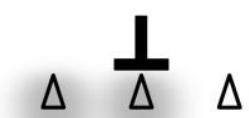

2

$\Delta \frac{1}{\Delta} \Delta$

4

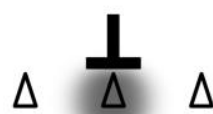

Figure 1: schematic sequence of ageing of a mobile edge dislocation by segregating solutes between two thermally activated jumps. (1) Dislocation aged to saturation within a remote solute concentration $\mathrm{C} 0$. (2) Instantaneous jump to the next row of obstacles. The flight time is infinitesimally small compared to solute diffusion time. (3) The segregated solute cloud at the initial position is redistributed in the uniform remote concentration field, while solutes locally segregate at the new dislocation position during the waiting time on the next obstacle. (4) After a sufficient time $t_{a} \gg \tau$ (see equation 2), the dislocation is aged again to saturation at its new position. If the remote concentration $\mathrm{C} 0$ is uniform, everything happens as if the solute cloud was "carried" by the dislocation from one arrest position to the next, whereas the actual process does not necessarily involve solute transport over such long distances. The situation may be different if hydrogen diffuses macroscopically from, e.g.: the left hand side, due to charging from a free surface.
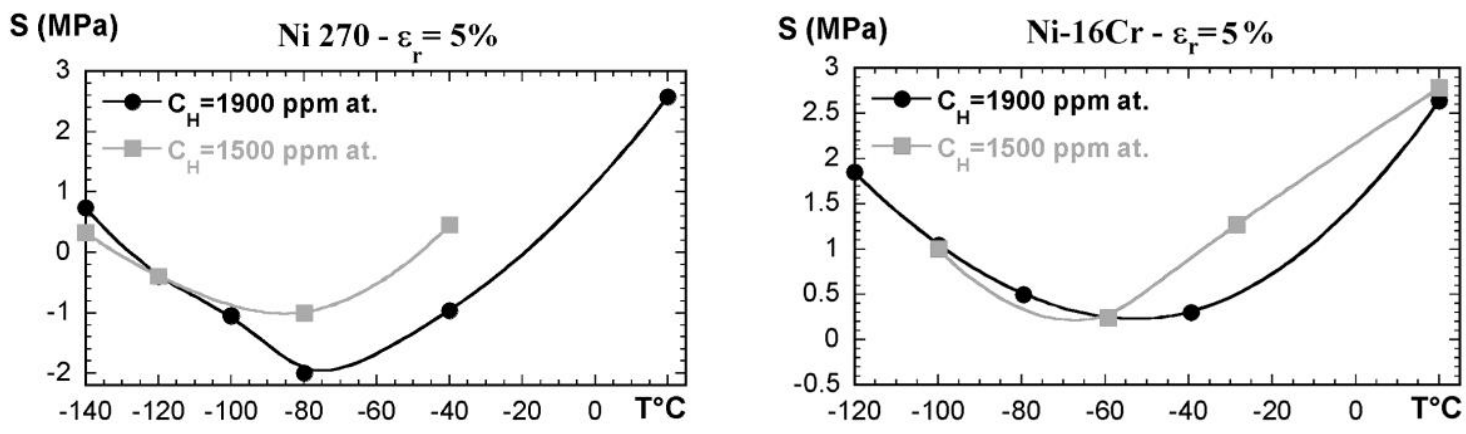

Figure 2: Strain Rate Sensitivity of hydrogenated (a) $\mathrm{Ni}$ and (b) $\mathrm{Ni}-\mathrm{Cr}$ as a function of temperature measured by strain rate jumps of amplitude 5 from a base strain rate of $10^{-}$ ${ }^{2} \mathrm{~s}^{-1}$ at $\varepsilon_{\mathrm{p}}=5 \%$ for both hydrogen contents. Both materials exhibit a minimum of SRS associated to DSA at cryogenic temperatures, but only Ni displays negative values that trigger PLC instabilities. 

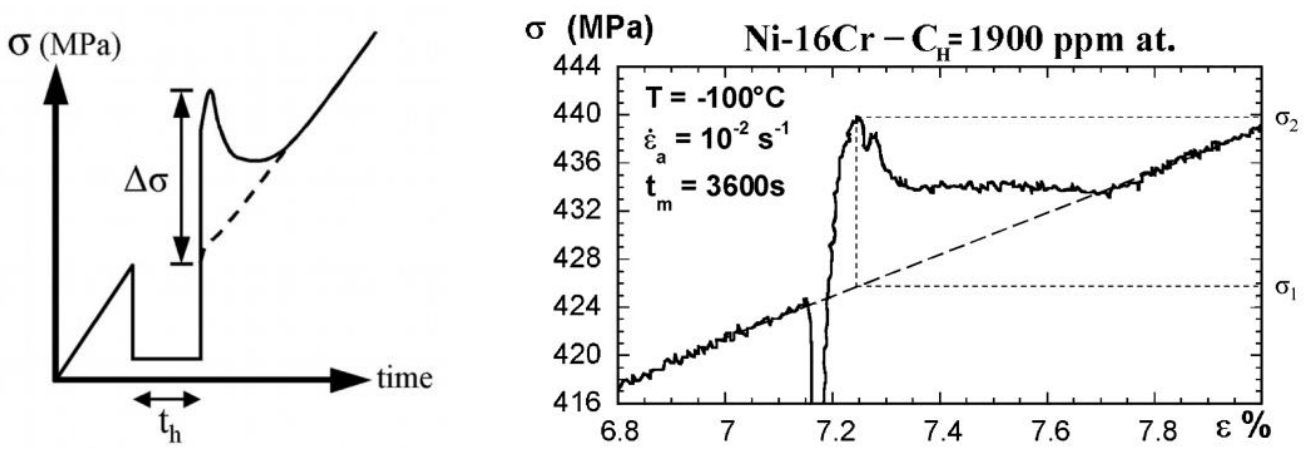

Figure 3: Static Strain Ageing experiments. The experimentally imposed holding time $t_{h}$ and ageing time $t_{a}$ are assumed equal (see text) (a) Schematic representation of the load transient after a holding time $t_{h}$ at near-zero applied stress. (b) measurement of $\Delta \sigma=\sigma_{2}-\sigma_{1}$ in Ni-16Cr containing 1600 at.ppm of hydrogen for $t_{h}=10 \mathrm{~h}$ at $-100^{\circ} \mathrm{C}$.
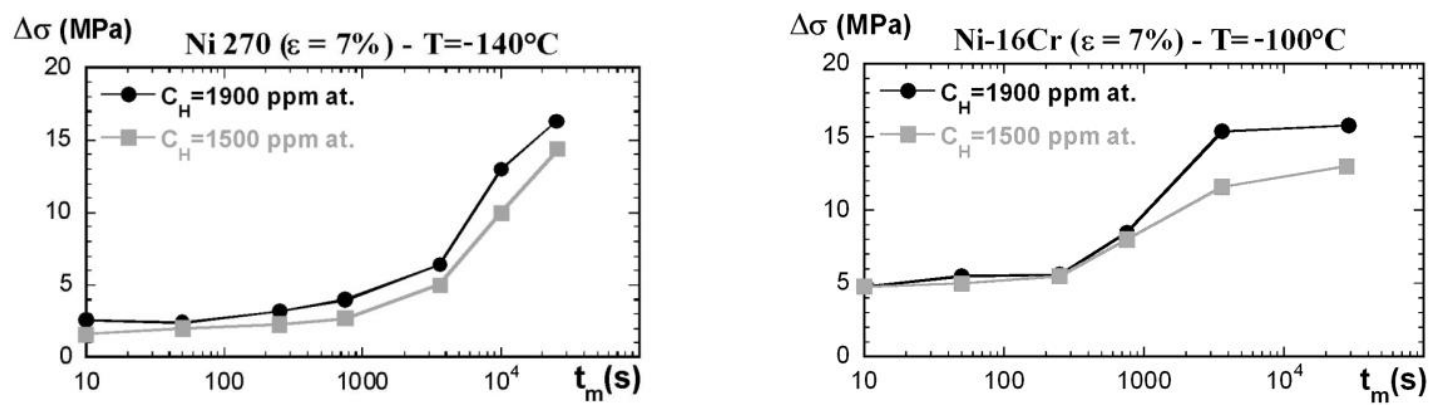

Figure 4: Static Strain Ageing peaks $(\Delta \sigma)$ measured as a function of the holding time $t_{h}$. (a) at $-100^{\circ} \mathrm{C}$ in $\mathrm{Ni}-16 \mathrm{Cr}$ and (b) at $-140^{\circ} \mathrm{C}$ in $\mathrm{Ni}$, for two hydrogen contents.

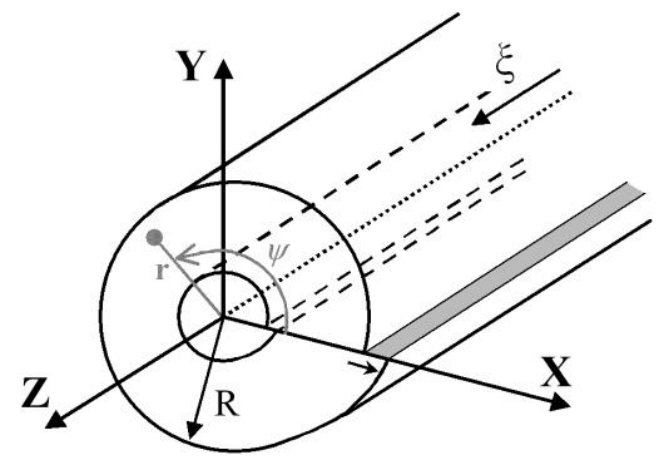

Figure 5: conventions for cylindrical coordinates around an edge dislocation line constructed by the classical Voltera procedure. 


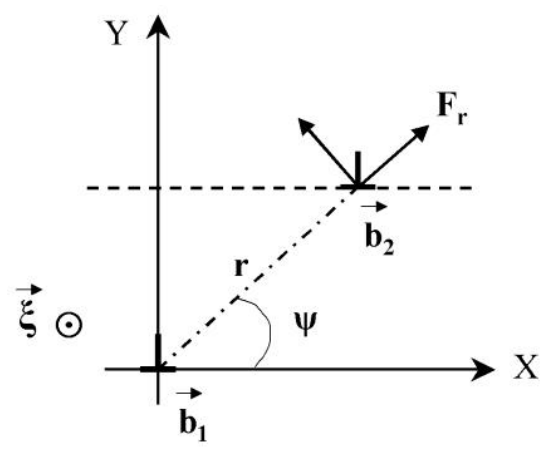

Figure 6: notation conventions for two interacting parallel edge dislocations

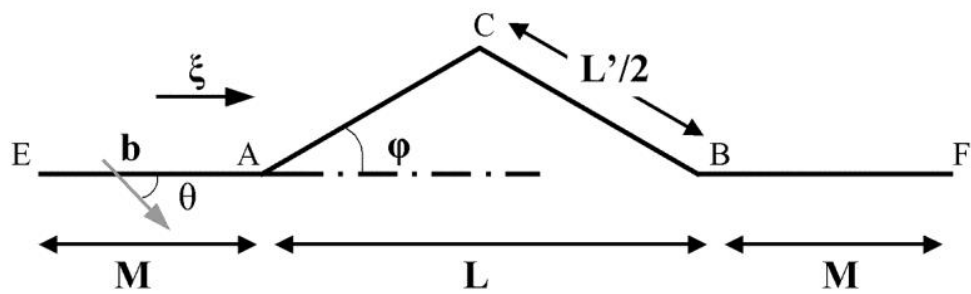

Figure 7: Incremental bow-out of length L from an originally straight segment, as modelled by Jøssang et al. (Jøssang et al. 1965) to evaluate the line tension. $b$ is Burger's vector and $\xi$ the unit vector of the dislocation line. The limit $\mathrm{M} \rightarrow \infty$ is used for calculations. Notations are consistent across both line tension models (equations 27 and 28)
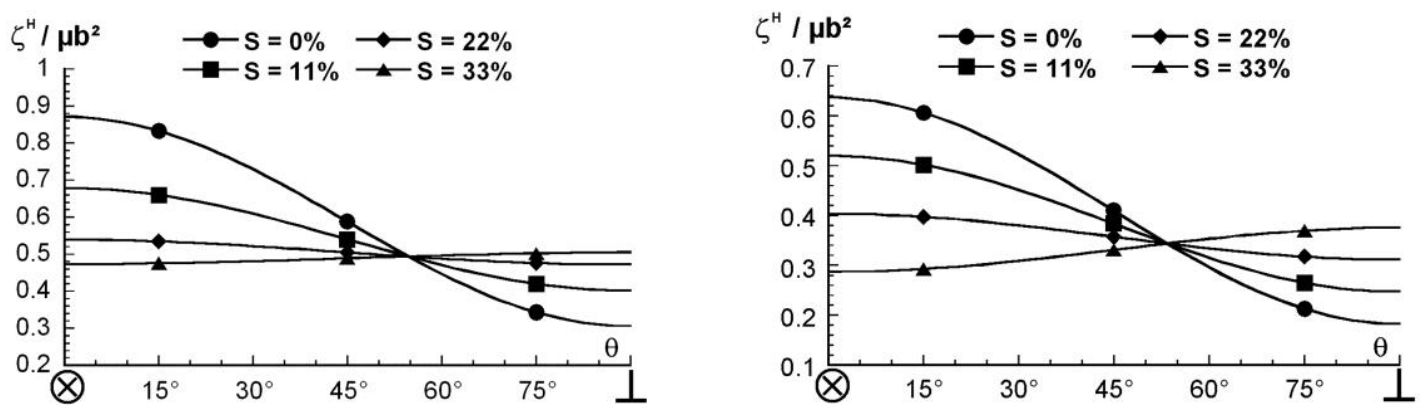

Figure 8: non dimensional value of the line tension (in units of $\mu b^{2}$ ) as a function of the dislocation character $\left(\theta=0^{\circ}\right.$ for screws), for four increasing values of the screening index S (T, c). (a) De Wit \& Koehler / Friedel model. (b) Jøssang / Hirth \& Lothe model. 

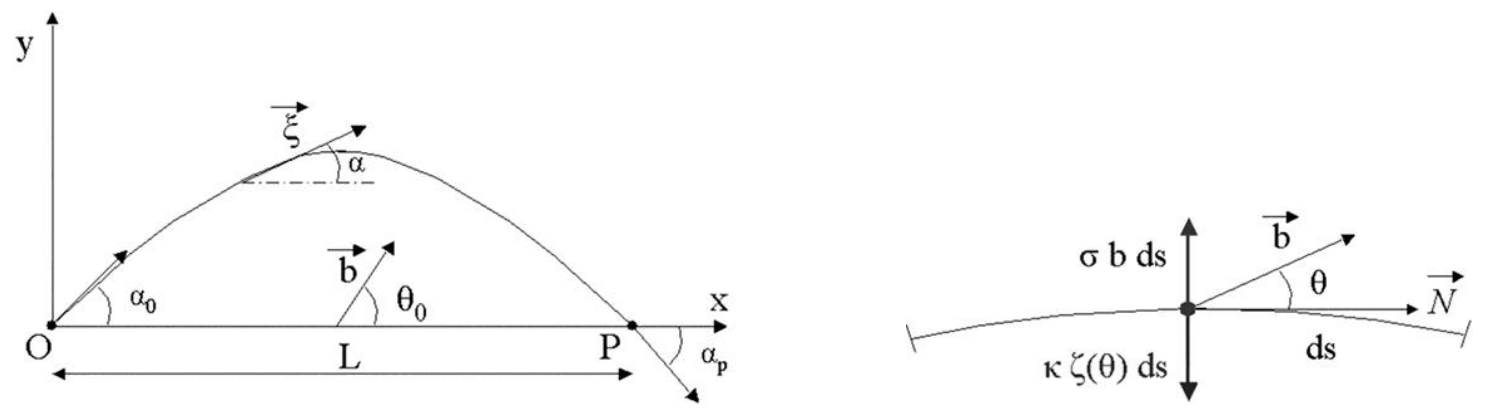

Figure 9: (a) geometry of a Franck-Read source. $b$ is Burger's vector, $\xi$ the line unit vector, and $\theta_{0}$ the character of the initially straight segment. (b) equilibrium of an elementary dislocation segment ds. The Peach-Koehler force $\sigma . b . d s$ counteracts the line tension $\kappa . \xi(\theta)$
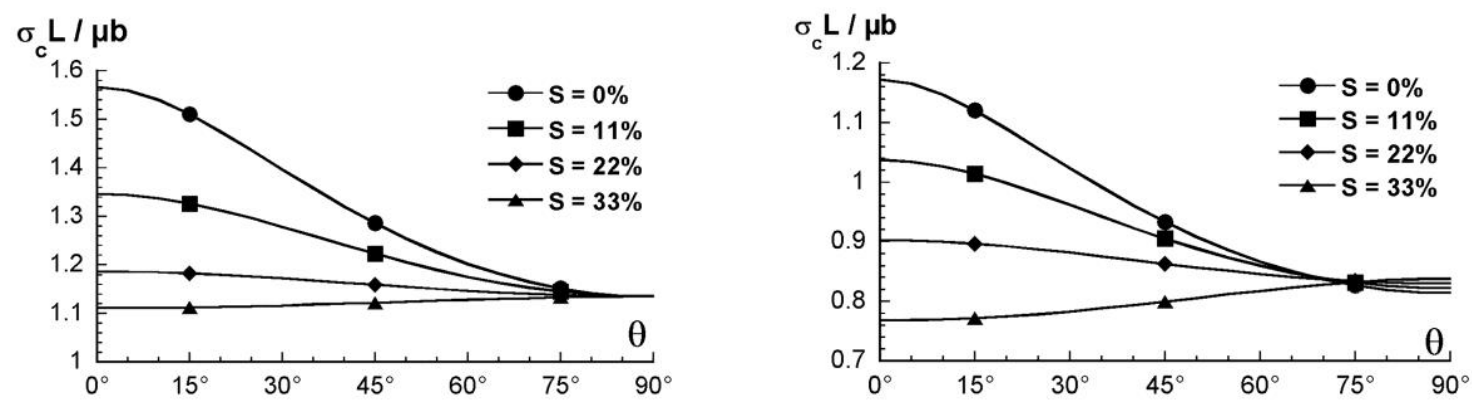

Figure 10: critical activation stress as a function of $S$ and $\theta_{0}$, here for $R / r_{0}=1250$. (a) De Wit \& Koehler / Friedel model. (b) Jøssang / Hirth \& Lothe model.

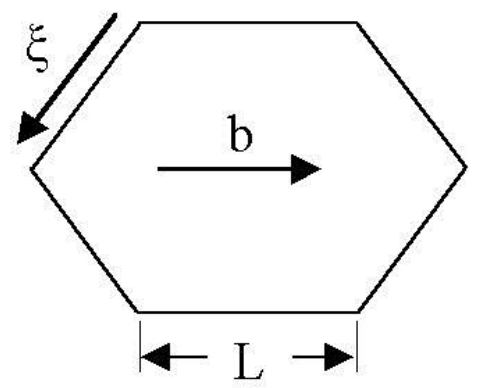

Figure 11: schematic of an hexagonal loop of side L, used as an approximation of a circular loop of radius $\mathrm{L}$ for the calculation of the line energy increase associated to isotropic loop expansion (after Hirth \& Lothe, 1992) 


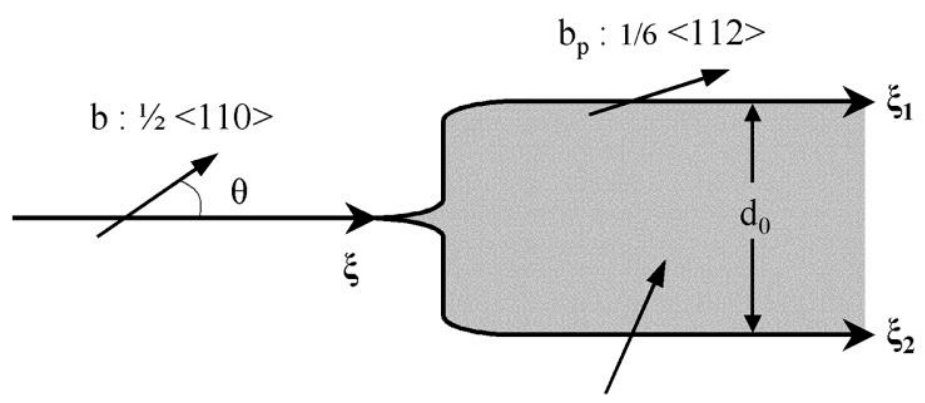

Figure 12: dissociation of a perfect dislocation of Burgers vector $b$, line vector $\xi$ and character $\theta$ in two Shockley partials $b_{1}$ and $b_{2}$ of Burgers vector $b_{p}$, line vector $\xi_{i}(i=1,2)$ and character $\theta+/-\pi / 6$ bounding a stacking Fault ribbon of width $\mathrm{d}_{0}$.

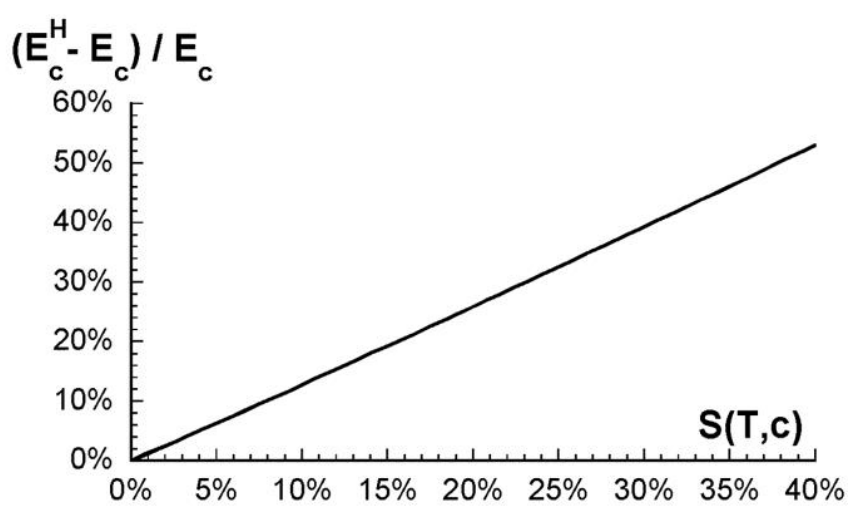

Figure 13: Relative increase of the energy barrier associated to the constriction of partial dislocations, as a function of the screening index $S$ (T, c), using the Püschl's semi-empirical relation (equation 40). A constant value of the SFE is assumed in this analysis, but $\Gamma$ is also liable to H-effects, which will add-up to the "elastic" contribution modelled here. 


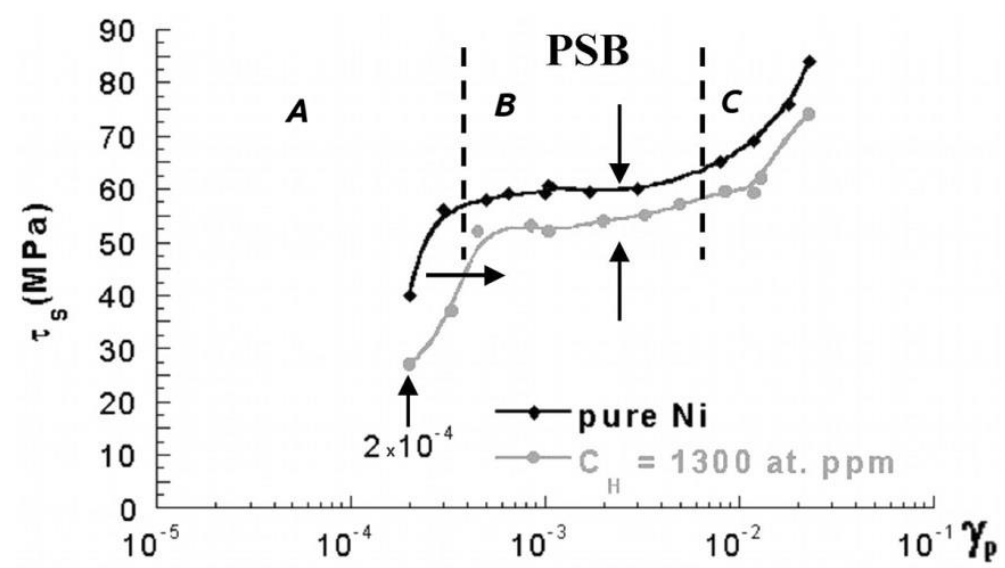

Figure 14: Cyclic stress-strain curves for $\mathrm{Ni}$ (black) and $\mathrm{Ni}-\mathrm{H}$ (grey) single crystals oriented for single glide. The collapse of the matrix-vein structure (region A) into a finer PSB structure (region B) is controlled by the cross-slip probability. Range of plastic strain amplitude $\Delta \varepsilon_{\mathrm{p}} / 2=0.2 \%$ to $2 \%$. Plastic strain rate: $10^{-3} \mathrm{~s}^{-1}$
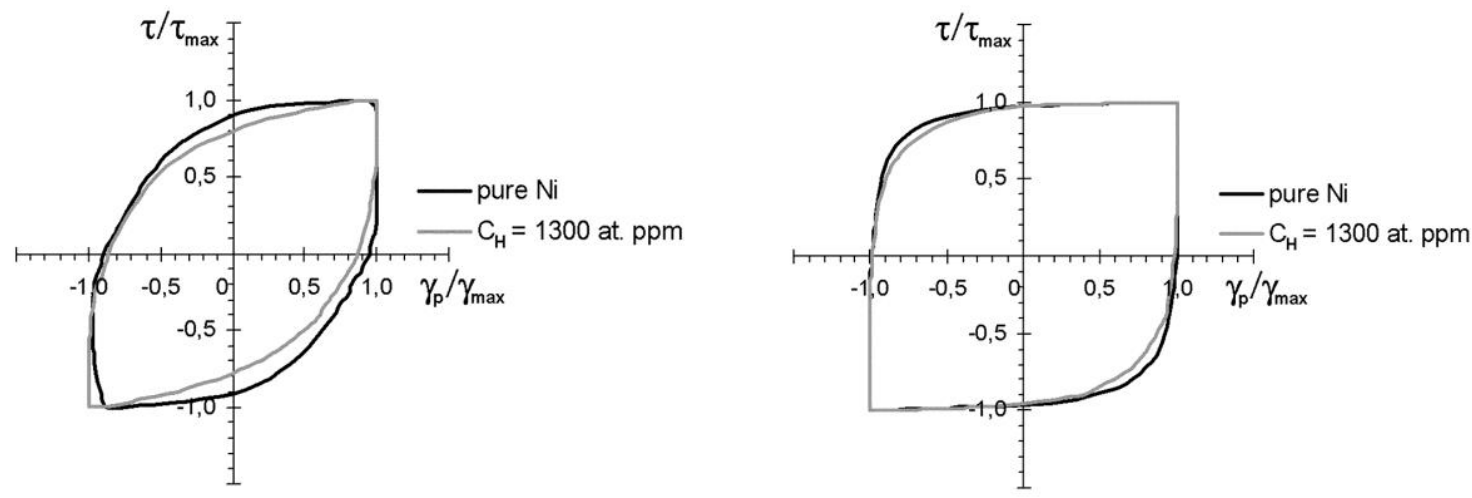

Figure 15: Normalized hysteresis stress-strain loops for Ni (black) and Ni-H (grey). (a) $\gamma_{\mathrm{p}}=1.210^{-3}$ amplitude. (b) $\gamma_{\mathrm{p}}=910^{-3}$ amplitude. Loops become more rectangular with increasing strain amplitude across the PSB domain. The delay at the onset of PSBs in $\mathrm{Ni}-\mathrm{H}$ is retained through the PSB region. 

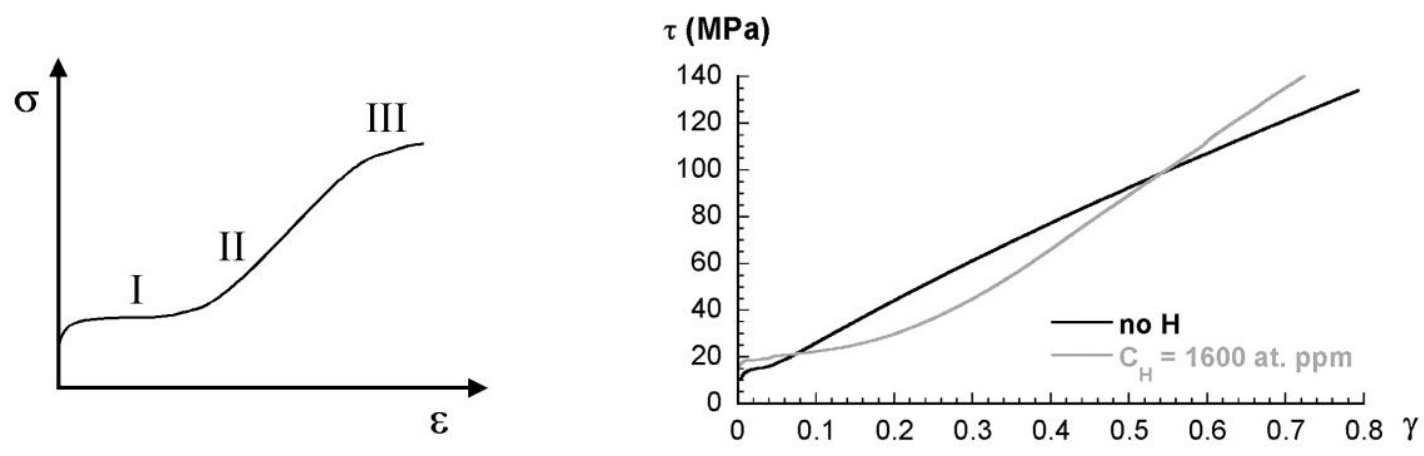

Figure 16: (a) schematic three-stage work hardening in single f.c.c. crystals oriented for single glide. (b) Experimental true stress-strain curves of non-charged and hydrogencharged nickel single crystals oriented for single slip. Shear stress normalised by $\mu$ the shear modulus
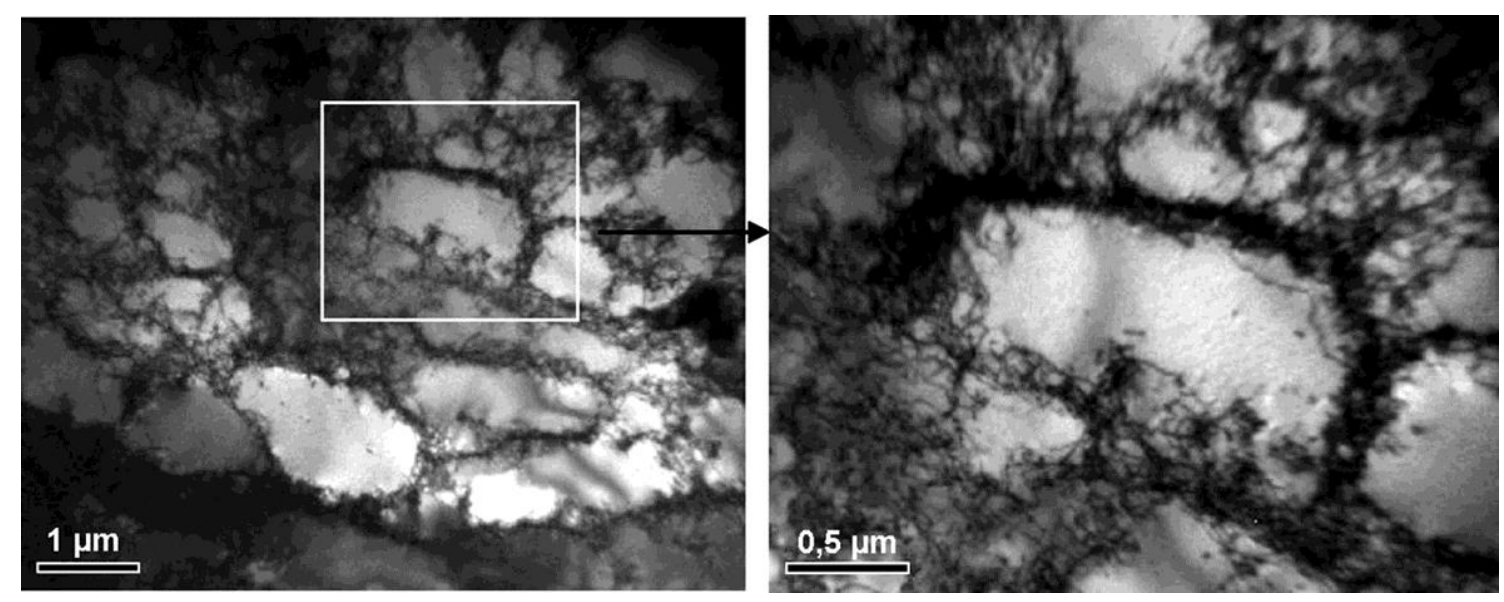

Figure 17: TEM observation of the dislocation structures in the [153] "single glide" plane of a Nickel single crystal at 0.75 plastic shear strain. The $\mathrm{Ni}$ crystal is well into the Stage III regime with the formation of equiaxed dislocation cells. 

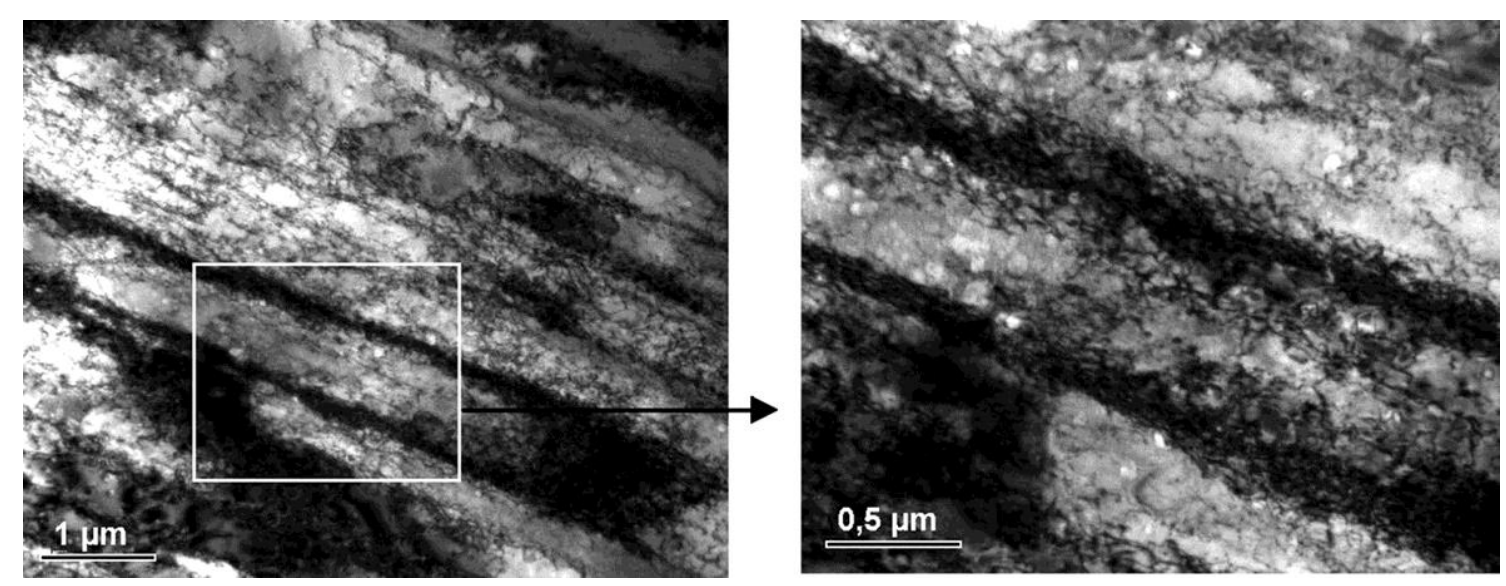

Figure 18: TEM observation of the dislocation structures in the [153] "single glide" plane of the hydrogenated Nickel single crystal at 0.75 plastic shear strain. A high density of planar dislocation walls is observed, typical of stage II deformation. The dislocation mean free path is significantly smaller in the case of pure $\mathrm{Ni}$ at the same deformation, causing a higher strain hardening rate in $\mathrm{Ni}-\mathrm{H}$. 